\title{
3D regularized $\mu(I)$-rheology for granular flows simulation
}

\author{
Alessandro Franci ${ }^{\mathrm{a}, *}$, Massimiliano Cremonesi ${ }^{\mathrm{b}}$ \\ ${ }^{a}$ International Center for Numerical Methods in Engineering (CIMNE) \\ ${ }^{b}$ Politecnico di Milano
}

\begin{abstract}
This paper proposes two regularized models of the $\mu(I)$-rheology and shows their application to the numerical simulation of 3D dense granular flows. The proposed regularizations are inspired by the Papanastasiou and Bercovier-Engleman methods, typically used to approximate the Bingham law. The key idea is to keep limited the value of the apparent viscosity for low shear rates without introducing a fixed cutoff. The proposed techniques are introduced into the Particle Finite Element Method (PFEM) framework to deal with the large deformations expected in free-surface granular flows. After showing the numerical drawbacks associated to the standard $\mu(I)$-rheology, the two regularization strategies are derived and discussed. The regularized $\mu(I)$-rheology is then applied to the simulation of the collapse of 2D and 3D granular columns. The numerical results show that the regularization techniques improve substantially the conditioning of the linear system without affecting the solution accuracy. A good agreement with the experimental tests and other numerical methods is obtained in all the analyzed problems.
\end{abstract}

\section{Introduction}

Dense granular flows are involved in several fields of engineering and technology such as geophysics, geotechnical engineering, mining, pharmaceutical and alimentary industry. Their understanding and modeling are therefore of paramount importance. Despite the intense research in both rheology and computational technology, the numerical simulation of granular materials is still challenging. Depending on the flow regime, granular materials can behave as solids, fluids or gases, and, in general, multiple phases may appear simultaneously. This behavior complicates the definition of their constitutive law as well as the identification of the most adequate technology for their numerical modeling.

\footnotetext{
* Corresponding author

Email addresses: falessandro@cimne.upc.edu (Alessandro Franci), massimiliano.cremonesi@polimi.it (Massimiliano Cremonesi)
} 
From the computational point of view, dense granular flows have been approached with both discrete and continuum methods. The Discrete Element Method (DEM) [17] is the most representative technique of the first family. A discrete approach simulates the granular flow by computing the interaction forces among each single particle. The explicit computation of contact forces and momentum enables a massive parallelized calculation and the solution of problems involving several millions of particles. Nevertheless, for the most of the industrial problems, this one-to-one approach is not feasible and a homogenization technique, such as the coarse graining techniques [2], is required. However, homogenization procedures are problem-dependent and require a calibration phase of the material parameters [58].

Conversely, continuum approaches describe the granular material as a unique body, regardless of the number of grains contained therein. However, in a continuum framework, the computational challenge becomes the definition of a constitutive model able to describe the different phases of the granular flow. Most of the models for granular flows are based on visco-plastic laws with the yield limit given by Drucker-Prager or Mohr-Coulomb type criterion [38, 16]. Very recently the so-called $\mu(I)$-rheology has been proposed for the simulation of dry and dense granular flows [43]. The model, originally proposed in a mono-dimensional framework, has been then extended to three-dimensional (3D) problems in [36], and applied for the first time to the granular column collapse simulation in [40].

The $\mu(I)$-rheology relates the shear stress to the pressure via a friction coefficient depending on the so-called inertial number $I$, a non-dimensional variable containing information of both microscopic and macroscopic scales. The model has several common points with both the Drucker-Prager [21] frictional law and the Bingham [7] non-Newtonian model. One of the main assumptions of the $\mu(I)$-rheology is to neglect the small volume variation observed in the dense regime of the granular flow [36]. This hypothesis, together with the direct relation between the shear stress and the shear strain rate, enables and facilitates its employment within a fluid-dynamics framework.

The simplicity of the implementation of the $\mu(I)$-rheology is counterbalanced by the limited number of application fields and by some numerical drawbacks. The $\mu(I)$-rheology assumes perfect rigid solid particles and a homogeneous flow. Hence, in principle, it should be applied only to steady and low sheared flows. Despite that, recent works, such as [39, 35], showed the applicability of the $\mu(I)$ rheology also to transient multi-directional 3D problems. This is also confirmed by the good agreement with the experimental collapse of granular columns of small aspect ratios shown in $[40,33,59]$. On the other hand, the method also suffers from numerical problems, such as the ill-conditioning of the algebraic solution system induced by the unbounded value of the apparent viscosity [55, 11] and the ill-posedness of the incompressible problem. The second numerical drawback has been already successfully approached in $[4,3,5]$, while a deep analysis of the first one is still missing.

The ill-conditioning generated by the model still represents a bottleneck for the application to real 3D cases. As already highlighted in [11], the stan- 
dard $\mu(I)$-rheology may lead to very ill-conditioned linear systems compromising the FEM solution. Furthermore, ill-conditioning forces the use of direct linear solvers [11], in general less performing than iterative ones for the solution of large systems. This also explains the lack of $3 \mathrm{D}$ applications of the $\mu(I)$ rheology in the literature. This work aims to shed light on the ill-conditioning problems of the constitutive model and to propose two regularized forms of the $\mu(I)$-rheology capable to reduce the ill-conditioning of the FEM solution. The models are inspired by the regularizations procedures of Bercovier-Engelman [6] and Papanastasiou [48] used to overcome similar numerical drawbacks of Bingham and Herschel-Bulkley [28] non-Newtonian laws. The regularized methods limit the value of the apparent viscosity when the shear strain rate tends to zero, approximating accurately the response of the standard $\mu(I)$-rheology and circumventing its endemic algebraic drawbacks. The proposed regularized models allow for the solution of large 3D problems using iterative linear solvers and maintaining limited the number of iterations.

Another typical issue associated to granular flow simulations is the large deformation suffered by the domain. Consequently, if mesh-based solvers are used, the tracking of the highly deforming contours can be difficult to be handled. On the other hand, particle methods can naturally deal with this issue and this explains their growing popularity in the context of granular flows simulations (e.g. $[12,24,29])$. In this work, mesh distortion issues are solved using the socalled Particle Finite Element Method (PFEM) [32]. The PFEM is a Lagrangian method that combines the solution of the governing differential equations with the Finite Element Method (FEM) with an efficient remeshing procedure [47]. The proposed regularizations have been implemented into a PFEM framework [45] enabling the solution of complex 3D free-surface problems. In this sense, this work wants also to give a further confirmation of the applicability of the PFEM to free-surface granular flows simulation, after the recent works $[19,60]$.

The paper is structured as follows. In Section 2 the main features of the $\mu(I)$ rheology are recalled. In Section 3, the numerical drawbacks of the standard $\mu(I)$-rheology are described and the two regularization procedures are derived and discussed. The balance equations as well as the solution scheme are presented in Section 4. In Section 5 the basic characteristics of the PFEM are recalled. Section 6 is dedicated to the $2 \mathrm{D}$ and $3 \mathrm{D}$ numerical simulation of the collapse of granular columns over horizontal and inclined planes. Finally, concluding remarks are given in Section 7.

\section{2. $\mu(I)$-rheology}

From the analysis of two-dimensional (2D) dense granular materials, [18] and [34] proposed the following relationship between shear stress $\tau$ and normal confinement pressure $p$

$$
\tau=\mu(I) p
$$


where the proportionality coefficient is a friction parameter $\mu$ which depends on the so-called inertial number $I^{1}$ defined as

$$
I=\frac{d_{g}}{\sqrt{p / \rho_{g}}} \dot{\gamma}
$$

where $d_{g}$ and $\rho_{g}$ are the grain diameter and density, respectively, and $\dot{\gamma}$ is the shear rate, obtained from the velocity $v_{x}$ as $\dot{\gamma}=\partial v_{x} / \partial y$. The inertial number can be seen as the ratio between a microscopic and a macroscopic characteristic time lengths. The first one, $\frac{d_{g}}{\sqrt{p / \rho_{g}}}$, refers to the particle scale, whereas the inverse of the shear strain rate $\frac{1}{\dot{\gamma}}$ represents the macroscopic time scale [36].

Relying on the experimental results presented in $[49,50],[36]$ proposed the following relation for the friction coefficient $\mu(I)$

$$
\mu(I)=\mu_{s}+\frac{\mu_{d}-\mu_{s}}{I_{0}+I} I
$$

where $I_{0}$ is a dimensionless material parameter and $\mu_{s}$ and $\mu_{d}$ are the static and the dynamic friction coefficients, respectively. The friction coefficient $\mu(I)$ tends to $\mu_{s}$ for very low values of the inertial number (and consequently of the shear strain rate), whereas it tends to $\mu_{d}$ for high values of $I$. An example of the typical behavior of $\mu(I)$ as a function of $I$ can be found in Figure 1.

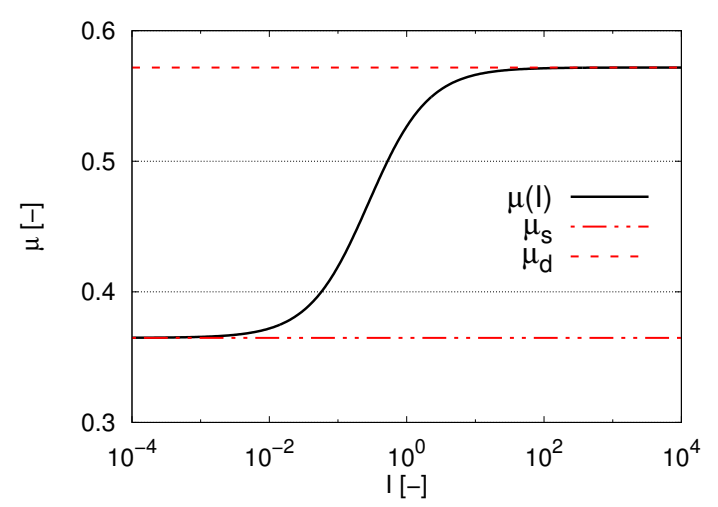

Figure 1: Relation between the friction coefficient $\mu(I)$ and the inertial number $I$ (material parameters from [36]: $\mu_{s}=0.3648, \mu_{d}=0.5718, \rho_{g}=2500 \mathrm{~kg} / \mathrm{m}^{3}, d_{g}=$ $1 \mathrm{~mm}$ and $I_{0}=0.279$, confinement pressure: $\left.100 \mathrm{~Pa}\right)$.

Eq.(1) holds only in a simplified 2D framework. The model proposed in [36] considers negligible the volume fraction variation in the dense regime, enabling the description of the granular material as an incompressible fluid. Considering this incompressibility hypothesis, the $\mu(I)$-rheology has been extended to a

\footnotetext{
${ }^{1}$ The inertial number $I$ can also be seen as the square root of the Savage number [56].
} 
three-dimensional (3D) fluid dynamic framework [36 ${ }^{2}$. In this scenario, it is useful to decompose the Cauchy stress tensor $\boldsymbol{\sigma}$ in its deviatoric and hydrostatic parts as

$$
\sigma_{i j}=-p \delta_{i j}+\tau_{i j}
$$

where $\tau_{i j}$ is the deviatoric stress, which is related to the deviatoric strain rate tensor $\dot{\gamma}_{i j}$ as

$$
\tau_{i j}=\eta \dot{\gamma}_{i j}
$$

where the apparent viscosity $\eta$ was defined in [36] as

$$
\eta=\frac{\mu(I) p}{|\dot{\gamma}|}
$$

being $|\dot{\gamma}|=\sqrt{\frac{1}{2} \dot{\gamma}_{i j} \dot{\gamma}_{i j}}$. In a 3D framework, the inertial number is defined as

$$
I=\frac{d_{g}}{\sqrt{p / \rho_{g}}}|\dot{\gamma}|
$$

Introducing Eq.(3) into Eq.(6), and calling $\Delta \mu=\mu_{d}-\mu_{s}$, the apparent viscosity can be rewritten as

$$
\eta=\left(\mu_{s}+\frac{\Delta \mu}{I_{0}+I} I\right) \frac{p}{|\dot{\gamma}|}
$$

and consequently, the shear stress - shear rate relationship can be written as

$$
\tau_{i j}=\left(\mu_{s}+\frac{\Delta \mu}{I_{0}+I} I\right) \frac{p}{|\dot{\gamma}|} \dot{\gamma}_{i j}
$$

Remark 1. As highlighted in [33, 11], negative apparent viscosity should be avoided. In [33], the pressure is imposed to be strictly positive in the apparent viscosity definition. On the other hand, [11] proposed an apposite regularization for the pressure to guarantee a positive value. Analogously, in this work, an arbitrary small positive pressure is always used in Eqs.(8-9) in tensile state. An alternative technique to guarantee a positive pressure based on unilateral incompressibility constraint has been proposed in [20].

\section{Regularized $\mu(I)$-rheology}

The shear stress definition of the $\mu(I)$-rheology (Eq.(9)) shows some analogies with both non-Newtonian fluid models and Drucker-Prager plasticity. In particular, as for the Bingham law, the $\mu(I)$-rheology describes a rigid-viscoplastic behavior where the material starts flowing only if a threshold is exceeded. Moreover, the stress limit $\left(\left|\tau_{i j}\right| \geq \mu_{s} p\right)$ is analogous to the Drucker-Prager condition. 


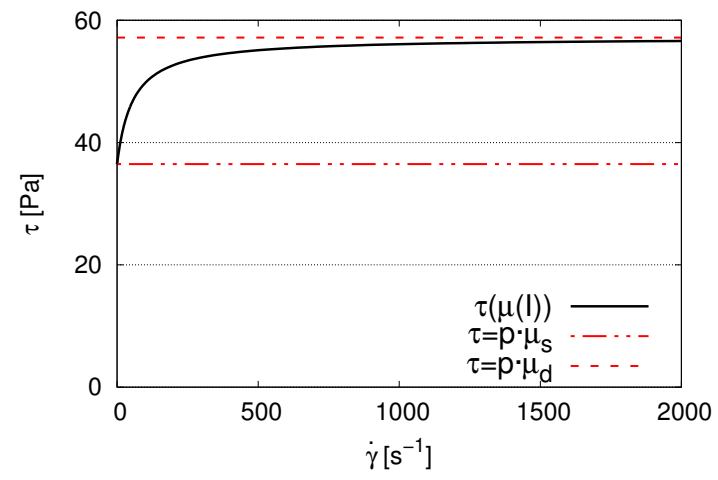

Figure 2: Relation between $\dot{\gamma}$ and $\tau$ (material parameters from [36]: $\mu_{s}=0.3648$, $\mu_{d}=0.5718, \rho_{g}=2500 \mathrm{~kg} / \mathrm{m}^{3}, d_{g}=1 \mathrm{~mm}$ and $I_{0}=0.279$, confinement pressure: 100 $\mathrm{Pa})$.

Figure 2 shows an example of the shear stress and strain rate relationship of the $\mu(I)$-rheology (Eq.(9)).

Figure 2 also shows that the shear stress is bounded between $\tau=p \mu_{s}$ and $\tau=p \mu_{d}$. On the contrary, the apparent viscosity (Eq.(8)) diverges to infinity when the shear strain rate tends to zero. For convenience, the apparent viscosity is decomposed in two contributions, $\eta_{s}$ and $\eta_{\Delta}$, the former depending on $\mu_{s}$ and the latter on $\Delta \mu$, as follows

$$
\eta=\eta_{s}+\eta_{\Delta}
$$

where

$$
\begin{gathered}
\eta_{s}=\frac{p \mu_{s}}{|\dot{\gamma}|} \\
\eta_{\Delta}=\frac{p \Delta \mu}{I_{0} \sqrt{p / \rho_{g}}+d_{g}|\dot{\gamma}|} d_{g}
\end{gathered}
$$

As shown in Figure 3, only the term $\eta_{s}$ diverges to infinity, whereas $\eta_{\Delta}$ tends to the finite value $\Delta \mu d_{g} \sqrt{p \rho_{g}} / I_{0}$. Furthermore, it is important to note that the component $\eta_{\Delta}$ is significant only for high values of $\dot{\gamma}$, while the term $\eta_{s}$ is predominant for small shear rates.

The jump between the rigid and the visco-plastic behavior in the stress function, and the divergent relation of the apparent viscosity, complicate significantly the implementation of the standard $\mu(I)$-rheology into a fluid dynamics framework and induce algebraic drawbacks, such as the ill-conditioning of the solution system. To overcome these inconveniences, two distinct regularizations of the $\mu(I)$-rheology are here proposed. Both methods are inspired by consolidated

\footnotetext{
${ }^{2}$ Note that the incompressibility of dense granular flow is not always a valid assumption. For example, in a silo discharge this hypothesis is not applicable to the region close to the aperture [35].
} 


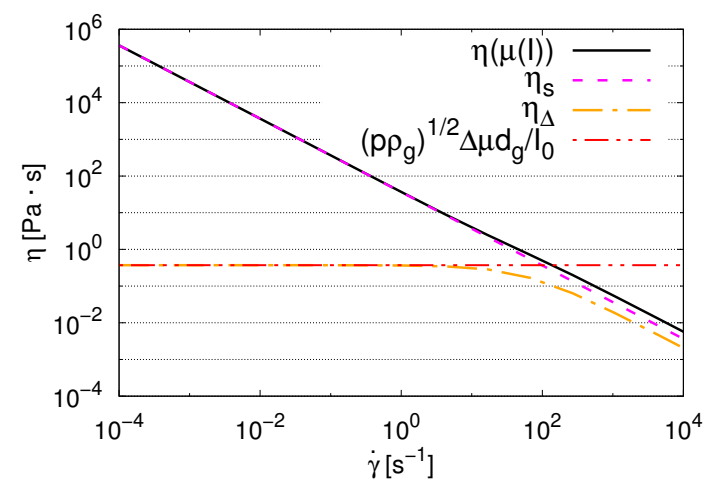

Figure 3: Relation between $\dot{\gamma}$ and $\eta$ (material parameters from [36]: $\mu_{s}=0.3648$, $\mu_{d}=0.5718, \rho_{g}=2500 \mathrm{~kg} / \mathrm{m}^{3}, d_{g}=1 \mathrm{~mm}$ and $I_{0}=0.279$, confinement pressure: 100 $\mathrm{Pa}$ ).

techniques used to regularize the Bingham non-Newtonian law. In particular, the Exponential regularization is inspired by the Papanastasiou [48] method, while the Penalty regularization is derived from the Bercovier-Engelman [6] model.

Remark 2. Both $\eta_{s}$ and $\eta_{\Delta}$ may become indeterminate when the shear strain rate and the pressure are simultaneously equal to zero. This condition may occur at the free-surface of a granular flow at rest [11]. However, from a numerical perspective, this situation could just occur at the beginning of the first computation, if both variables are initialized at zero. For the rest of the analysis, the pressure and the shear strain rate can get close to nullify but they will be never exactly equal to zero, if they are not prescribed in a strong form. Hence, this undesired eventuality can be circumvented with a simple initialization of the model variables. This explains why the proposed regularization techniques are not designed to avoid this numerical inconvenience.

It is important to mention that similar regularizations were already proposed in [11] and [10] and subsequently used in practical problems [52, 1]. However, while in [11] both terms $\eta_{s}$ and $\eta_{\Delta}$ were modified, in this work the regularization is applied only to $\eta_{s}$, which is the term responsible of the divergent behavior of the $\mu(I)$-rheology.

The proposed regularized forms of $\eta_{s}$ according to the Exponential and the Penalty methods read respectively

$$
\eta_{s}^{E}=\frac{p \mu_{s}}{|\dot{\gamma}|}\left(1-e^{-\frac{|\dot{\gamma}|}{\lambda}}\right)
$$

and

$$
\eta_{s}^{P}=\frac{p \mu_{s}}{\sqrt{|\dot{\gamma}|^{2}+\lambda^{2}}}
$$


where $\lambda$ is a regularization parameter. Note that both methods recover the original form of Eq.(11) for $\lambda=0 s^{-1}$.

In conclusion, the regularized apparent viscosities according to the Exponential $\left(\eta^{E}\right)$ and Penalty $\left(\eta^{P}\right)$ strategies are computed respectively as

$$
\eta^{E}=\eta_{s}^{E}+\eta_{\Delta}
$$

and

$$
\eta^{P}=\eta_{s}^{P}+\eta_{\Delta}
$$

Note that the limit of both $\eta^{E}$ and $\eta^{P}$ for the shear rate going to zero, is

$$
\lim _{|\dot{\gamma}| \rightarrow 0} \eta^{E}=\lim _{|\dot{\gamma}| \rightarrow 0} \eta^{P}=\frac{p \mu_{s}}{\lambda}+\frac{\Delta \mu d_{g} \sqrt{p \rho_{g}}}{I_{0}}
$$

Figure 4 shows the approximation of the apparent viscosity given by Eq.(15) and Eq.(16), considering three different values of the regularization parameter $\lambda$, while Figures 5 and 6 plot the resulting $(\tau, \dot{\gamma})$ curve for the Exponential and the Penalty methods, respectively.

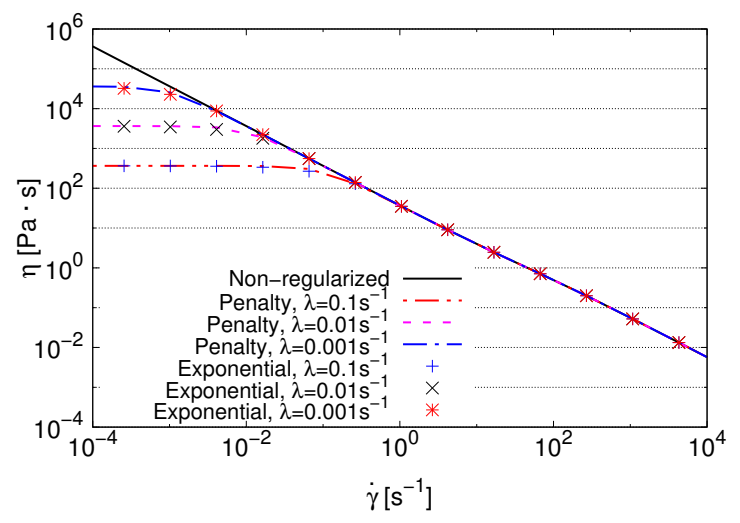

Figure 4: Relation between $\dot{\gamma}$ and $\eta$ for the non-regularized model and the Exponential and Penalty regularizations with three different values of stabilization parameter $\lambda$.

The graphs show that both Exponential and Penalty methods give an accurate approximation of the $(\tau, \dot{\gamma})$ curve of the standard $\mu(I)$-rheology. The main differences appear for small shear strain rates, as highlighted in Figures 5b and 6b. For $\dot{\gamma}=0 s^{-1}$, the non-regularized curve tends to $p \mu_{s}$, whereas the regularized curves tend to zero with a slope depending on the value of the regularization parameters. As expected, the smaller is the stabilization parameter $\lambda$, the better is the approximation given by the regularized curves. The graphs also show that the Penalty and Exponential approximations give an almost identical response when they use the same $\lambda$. 
(a)

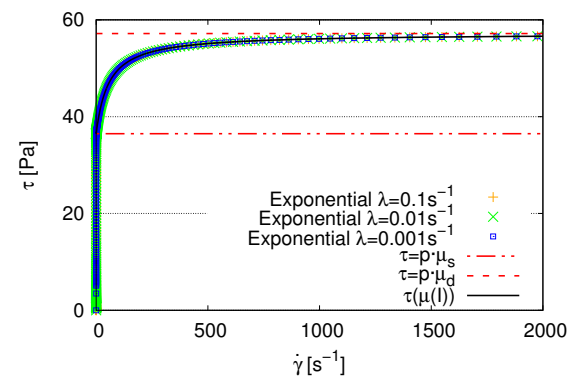

(b)

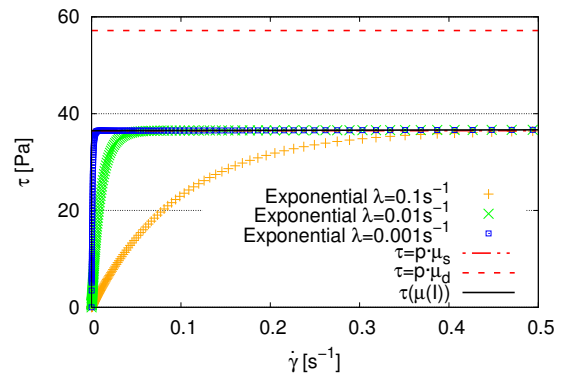

Figure 5: (a) Relation between $\dot{\gamma}$ and $\tau$ for the non-regularized model and Exponential regularization with three different values of stabilization parameter $\lambda$; (b) Enlargement for very low $\dot{\gamma}$.

Remark 3. In this non-linear model, the apparent viscosity $\eta$ does not coincide with the derivative (tangent) of the $(\tau, \dot{\gamma})$ curve. In fact, for the monodimensional case, the slope of the $(\tau, \dot{\gamma})$ curve is given by

$$
\frac{\partial \tau}{\partial \dot{\gamma}}=\frac{\partial \eta}{\partial \dot{\gamma}} \dot{\gamma}+\eta \frac{\partial \dot{\gamma}}{\partial \dot{\gamma}}=\frac{\partial \eta}{\partial \dot{\gamma}} \dot{\gamma}+\eta
$$

A graphical check can be done by comparing Figure 4 with Figures 5 and 6.

\section{Balance equations and solution strategy}

The regularized $\mu(I)$-rheology has been introduced into a computational fluid dynamics framework. The motion of the granular material is governed by the Navier-Stokes equations. Momentum and mass conservations are written in the current domain $\Omega_{t}$. Following $[31,53,26]$, in the mass conservation equation a small compressibility is admitted through the material bulk modulus. So, the differential governing equations are

$$
\begin{gathered}
\rho \dot{\boldsymbol{v}}-\nabla \cdot \boldsymbol{\sigma}-\rho \mathbf{b}=\mathbf{0} \quad \text { in } \Omega_{t} \times(0, T) \\
\frac{1}{\kappa} \dot{p}+\nabla \cdot \boldsymbol{v}=0 \quad \text { in } \Omega_{t} \times(0, T)
\end{gathered}
$$

where $\boldsymbol{v}$ is the velocity field, $\rho$ is the density of the bulk, $\mathbf{b}$ are the body forces, and $\kappa$ is the bulk modulus. Note that for $\kappa \rightarrow \infty$ the fully-incompressible form of the continuity equation $(\nabla \cdot \boldsymbol{v}=0)$ is recovered. In the numerical tests presented in this work $\kappa=10^{9} \mathrm{~Pa}$ has been considered. The problem Eqs.(19)-(20) has to be supplemented with appropriate initial and boundary conditions.

The balance equations are discretized according to the standard Galerkin Finite Element Method (FEM). In particular, the velocity-pressure stabilized 
(a)

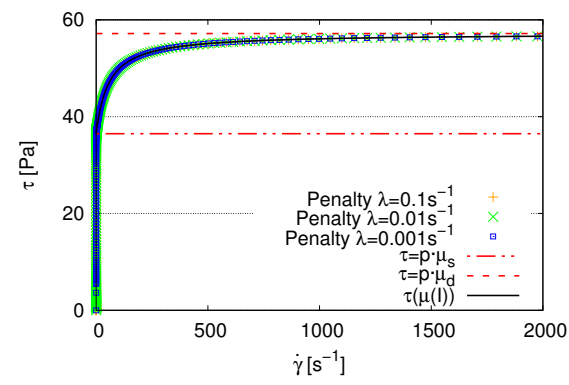

(b)

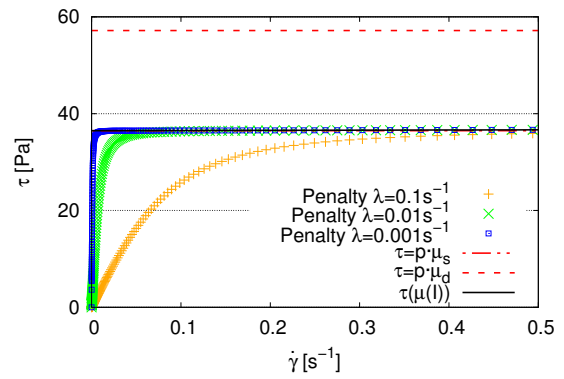

Figure 6: (a) Relation between $\dot{\gamma}$ and $\tau$ for the non-regularized model and Penalty regularization with three different values of stabilization parameter $\lambda$; (b) Enlargement for very low $\dot{\gamma}$.

scheme proposed and validated for Newtonian fluids simulations in [45] is used. The Finite Calculus (FIC) stabilization $[44,45,51]$ is adopted to stabilize spurious oscillations due to linear interpolation used for both the velocity and pressure unknowns [8]. The derivation of the FEM solution scheme is out of the scope of this work and only the final fully-discretized and linearized solution scheme is given (all the details can be found in [45]).

At each time step $\left[{ }^{n} t ;{ }^{n+1} t\right]$ of duration $\Delta t$, the linear momentum and the mass balance equations are solved iteratively for velocity increments and pressure, as described below.

At each non-linear iteration $i$ :

1. Compute the nodal velocity increments $\Delta \overline{\boldsymbol{v}}$ :

$\boldsymbol{K}^{i} \boldsymbol{\Delta} \overline{\boldsymbol{v}}=\boldsymbol{R}^{i}\left(\overline{\boldsymbol{v}}^{i}, \overline{\boldsymbol{p}}^{i}\right)$

where $\boldsymbol{K}^{i}=\boldsymbol{K}^{m}\left(\overline{\boldsymbol{x}}^{i}, \boldsymbol{c}\right)+\boldsymbol{K}^{\rho}\left(\overline{\boldsymbol{x}}^{i}\right)$

being $\overline{\boldsymbol{x}}^{i}$ the nodal coordinates.

2. Update the nodal velocities: ${ }^{n+1} \overline{\boldsymbol{v}}^{i+1}={ }^{n+1} \overline{\boldsymbol{v}}^{i}+\boldsymbol{\Delta} \overline{\boldsymbol{v}}$

3. Update the nodal coordinates: ${ }^{n+1} \overline{\boldsymbol{x}}^{i+1}={ }^{n+1} \overline{\boldsymbol{x}}^{i}+\overline{\boldsymbol{u}}(\boldsymbol{\Delta} \overline{\boldsymbol{v}})$ being $\overline{\boldsymbol{u}}$ the nodal displacements.

4. Compute the nodal pressures $\overline{\boldsymbol{p}}^{i+1}: \boldsymbol{H} \overline{\boldsymbol{p}}^{i+1}=\boldsymbol{F}_{p}\left(\overline{\boldsymbol{v}}^{i+1}, \overline{\boldsymbol{p}}^{i}\right)$

where $\boldsymbol{H}=\left(\frac{1}{\Delta t} \mathbf{M}_{1}+\frac{1}{\Delta t^{2}} \mathbf{M}_{2}+\mathbf{L}+\mathbf{M}_{b}\right)$

and $\boldsymbol{F}_{p}=\frac{\mathbf{M}_{1} n}{\Delta t} \overline{\boldsymbol{p}}+\frac{\mathbf{M}_{2}}{\Delta t^{2}}\left({ }^{n} \overline{\boldsymbol{p}}+{ }^{n} \overline{\dot{\boldsymbol{p}}} \Delta t\right)-\mathbf{Q}^{T} \overline{\boldsymbol{v}}^{i+1}+\boldsymbol{f}_{p}$

5. Compute the Cauchy stresses: $\boldsymbol{\sigma}^{i+1}$

6. Check the convergence: $\frac{\left\|\Delta \overline{\boldsymbol{v}}^{i+1}\right\|}{\left\|^{n} \overline{\boldsymbol{v}}\right\|} \leq e_{v}, \frac{\left\|\overline{\boldsymbol{p}}^{i+1}-\overline{\boldsymbol{p}}^{i}\right\|}{\left\|^{n} \overline{\boldsymbol{p}}\right\|} \leq e_{p}$

with $e_{v}$ and $e_{p}$ prescribed error norms for velocities and pressures.

If condition 6 is not fulfilled, return to 1 with $i \leftarrow i+1$.

Note that the variables marked with a upper bar are nodal values. The vectors and matrices introduced in the previous list are given in Box 1. 


$$
\begin{aligned}
& R_{I i}^{i}=\int_{\Omega} N_{I} \rho N_{J} d \Omega \overline{\dot{v}}_{J i}^{i}+\int_{\Omega} \frac{\partial N_{I}}{\partial x_{j}} \sigma_{i j}^{i} d \Omega-\int_{\Omega} N_{I}^{n+1} b_{i} d \Omega \\
& \boldsymbol{K}_{I J}^{m}=\int_{\Omega} \boldsymbol{B}_{I}^{T} \boldsymbol{c} \boldsymbol{B}_{J} d \Omega, \boldsymbol{K}_{I J}^{\rho}=\boldsymbol{I} \int_{\Omega} N_{I} \frac{2 \rho}{\Delta t} N_{J} d \Omega, \mathbf{Q}_{I J}=\int_{\Omega} \mathbf{B}_{I}^{T} \mathbf{m} N_{J} d \Omega \\
& M_{1_{I J}}=\int_{\Omega} \frac{1}{\kappa} N_{I} N_{J} d \Omega, M_{2_{I J}}=\int_{\Omega} \tau \frac{\rho}{\kappa} N_{I} N_{J} d \Omega, \tau=\left(\frac{8 \eta}{h^{2}}+\frac{2 \rho}{\delta}\right)^{-1} \\
& M_{b_{I J}}=\int_{\Gamma_{t}} \frac{2 \tau}{h_{n}} N_{I} N_{J} d \Gamma, L_{I J}=\int_{\Omega} \tau\left(\nabla^{T} N_{I}\right) \nabla N_{J} d \Omega \\
& f_{p_{I}}=\int_{\Gamma_{t}} \tau N_{I}\left[\rho \frac{D v_{n}}{D t}-\frac{2}{h_{n}}\left(2 \eta d_{n}-t_{n}\right)\right] d \Gamma-\int_{\Omega} \tau \nabla^{T} N_{I} \boldsymbol{b} d \Omega
\end{aligned}
$$

where $\Gamma_{t}$ are the free-surface contours of the domain, $N_{I}$ are the linear shape functions and $h$ and $\delta$ are characteristic distances in space and time [45].

For 2D problems:

$$
\boldsymbol{c}=\left[\begin{array}{ccc}
\kappa \Delta t+\frac{4 \eta}{3} & \kappa \Delta t-\frac{2 \eta}{3 t} & 0 \\
\kappa \Delta t-\frac{2 \eta}{3} & \kappa \Delta t+\frac{4 \eta}{3} & 0 \\
0 & 0 & \eta
\end{array}\right], \mathbf{B}_{I}=\left[\begin{array}{cc}
\frac{\partial N_{I}}{\partial x} & 0 \\
0 & \frac{\partial N_{I}}{\partial y} \\
\frac{\partial N_{I}}{\partial y} & \frac{\partial N_{I}}{\partial x}
\end{array}\right], \boldsymbol{m}=\left[\begin{array}{l}
1 \\
1 \\
0
\end{array}\right]
$$

\section{Box 1}

Remark 4. The non-linearity of problem Eqs.(19)-(20) arises not only from large displacement and deformation hypothesis but also from the non-constant value of the apparent viscosity $\eta$ which is computed according the $\mu(I)$ constitutive law.

\section{Particle Finite Element Method (PFEM)}

The Particle Finite Element Method (PFEM) is a fully Lagrangian numerical technique that combines the standard FEM with an efficient remeshing algorithm. The PFEM has been used in a wide range of fields, including freesurface fluid dynamics [32, 45, 9], fluid-structure interaction [31, 15, 27], bed erosion [46], compressible flows [14], landslides [13] and granular flows [61].

With the PFEM the governing differential equations are integrated over the current discretized computational domain as in standard FEM. However, when the mesh becomes too distorted, the element connectivities are erased and a new mesh is built over the updated nodes positions via a two-step algorithm. First, a Delaunay triangulation [23] is performed with the aim of improving the quality of the simplices of the previous distorted mesh. Then, the so-called Alpha Shape method [22] is applied to detect the physical contours of the computational 
domain via a geometrical check. The details and implications of the PFEM remeshing procedure can be found in [30, 47, 25].

The basic step of the PFEM solution algorithm are summarized below.

1. Solve the non-linear governing equations of motion.

2. Update the mesh nodes positions.

3. Check the mesh distortion.

If the mesh is distorted:

(a) Erase the elements of the previous mesh (Figure 7a).

(b) Perform the Delaunay triangulation (Figure 7b).

(c) Do the Alpha Shape check (Figure 7c).

4. Go ahead to the next time step.

(a)

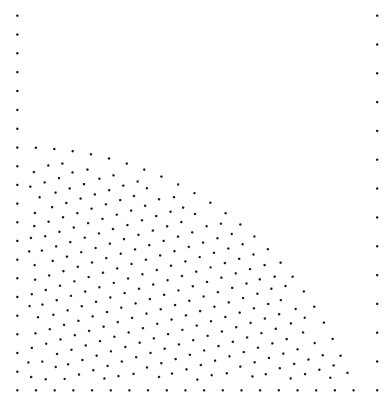

(b)

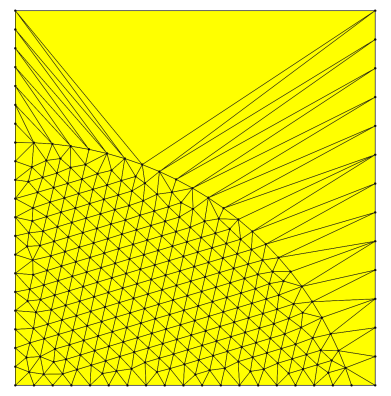

(c)

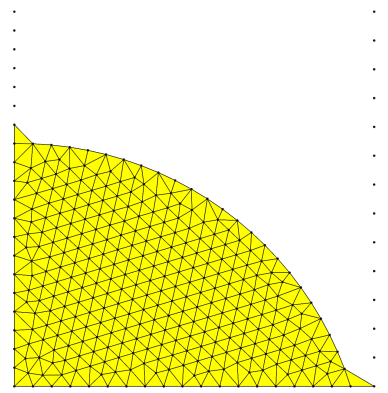

Figure 7: PFEM remeshing algorithm.

The continuous elimination of the mesh and the consequent loss of information stored at the Gauss points, make the PFEM more appropriate to formulations that do not require the storage of historical variables. From this perspective, the $\mu(I)$-rheology, enabling to treat the dense granular material as a fluid with an apparent viscosity, is particularly attractive for a PFEM framework.

\section{Numerical examples}

The proposed regularized models of the $\mu(I)$-rheology are here applied to the simulation of three different problems. The first numerical test is the collapse of the granular mass of small aspect ratio over an horizontal plane presented in [59] and simulated in the same work using the $\mu(I)$-rheology. This $2 \mathrm{D}$ problem is used to highlight the beneficial effect given by the regularized methods, to compare the Exponential and the Penalty strategies and to highlight the effect of the regularization parameter $\lambda$. Then, the granular flow over a plane inclined with an angle of $16^{\circ}$ is analyzed considering the real 3D geometry of the experimental setup [42]. The flow of the same granular material is then studied for other three different inclinations of the plane. The numerical results are 
compared to the experimental data [42] and the numerical results of [33]. To show the applicability of the proposed method to real 3D problems, the collapse of a cylindrical column of granular material of high aspect ratio is also studied. In this case, the PFEM results are compared to those obtained with a DEM formulation [39]. In all the numerical tests, no-slip boundary conditions have been considered for the nodes belonging to the rigid walls and the interaction with the surrounding air has not been taken in consideration.

\subsection{Granular column collapse}

The collapse of a glass beads column of height $H=50 \mathrm{~mm}$ and width $2 L=80 \mathrm{~mm}$ is induced by removing the vertical lateral walls (see Figure 8). The lifting velocity of the lateral walls is not provided in the reference work [59]. After analyzing the experimental results shown in [59], a constant lifting velocity $v_{L}=0.38 \mathrm{~m} / \mathrm{s}$ is here considered. The glass beads have diameter $d_{g}=2.0 \mathrm{~mm}$ and the following material properties: $\rho_{g}=2500 \mathrm{~kg} / \mathrm{m}^{3}$, $\mu_{s}=\tan \left(20.9^{\circ}\right), \mu_{d}=\tan \left(32.76^{\circ}\right)$ and $I_{0}=0.279$ [59]. The bulk density is kept fixed to $\rho=1450 \mathrm{~kg} / \mathrm{m}^{3}$. As reported in [59], the experiments were carried out in a narrow channel and a two-dimensional (2D) behavior was noted, justifying the $2 \mathrm{D}$ simulations here proposed.

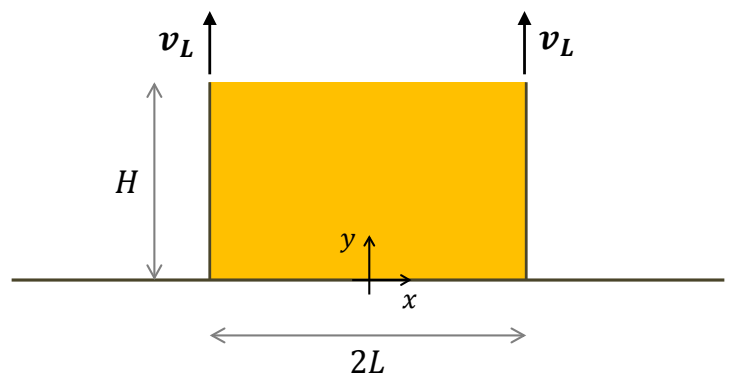

Figure 8: Granular column collapse. Initial geometry.

The example is solved with four different mesh sizes, namely $0.005 \mathrm{~m}, 0.003 \mathrm{~m}$, $0.001 \mathrm{~m}$ and $0.00075 \mathrm{~m}$, leading to $338,1006,9054$ and 16324 triangular elements, respectively. All the analyses are run for a global duration of $1 s$.

First, the numerical results obtained with the Exponential regularization with $\lambda=0.01 s^{-1}$ and the finest mesh are validated against the experimental data. Then, a deep analysis of the regularization effects is performed.

\subsubsection{Validation}

The numerical and experimental results obtained at three different time instants are shown in Figure 9. The reference time is given considering the dimensionless variable $t^{*}=t / \sqrt{g / H}$, where $g=9.81 \mathrm{~m} / \mathrm{s}^{2}$ is the gravity acceleration.

Figure 10 shows the free-surface position obtained numerically and experimentally at $t^{*}=5.88$, when the granular column has reached the rest configuration. Also the results obtained in [59] with a SPH method and the $\mu(I)$-rheology are plotted. 
(a) Numerical results at $t^{*}=2.09$

(b) Experimental results at $t^{*}=2.09$

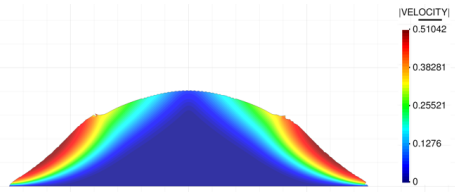

(c) Numerical results at $t^{*}=2.93$

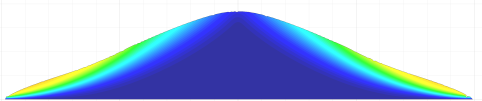

(e) Numerical results at $t^{*}=3.77$

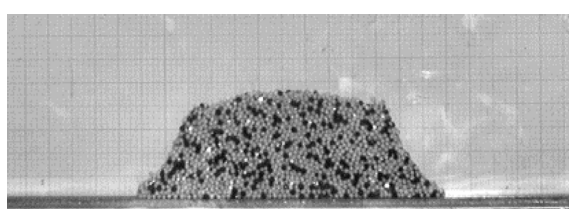

(d) Experimental results at $t^{*}=2.93$

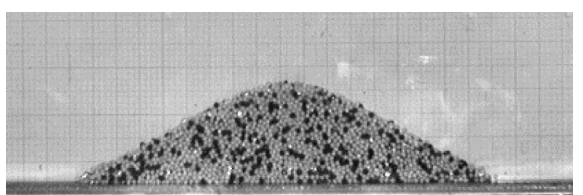

(f) Experimental results at $t^{*}=3.77$

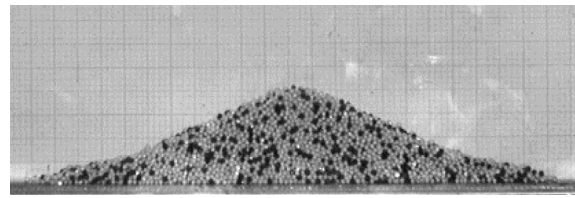

Figure 9: Granular column collapse. Numerical and experimental [59] results at three time instants. Colors represent the velocity magnitude.

Figures 9 and 10 show a general good agreement between the numerical solutions and the experimental results, although there are some discrepancies. The differences are more evident at the initial phase of the runout. These may be produced by the not accurate modeling of the experimental lifting wall motion, which, as already mentioned, was not provided in the reference work [59]. With respect to the final configuration (Figure 10), the numerical results give a slightly higher slump of the granular column (the final height is smaller) and a reduced spread (the predicted maximum horizontal displacement is smaller than the experimental one). These results are in accordance with the 2D SPH numerical results of $[59]$.

\subsubsection{Analysis of regularization methods}

To test the efficiency of the proposed regularized $\mu(I)$-rheology models approaches, the conditioning of the linear system, which arises from the finite element discretization, is here monitored. An index of the quality of the system conditioning is the number of the iterations required by an iterative linear solver to converge ${ }^{3}$. The worse conditioned is the linear system, the higher is the number of iterations of the solver. Based on that, the number of iterations is here considered as a measure of the linear system conditioning.

\footnotetext{
${ }^{3}$ Typically, for large scale fluid problems, iterative solvers are much more performing with respect to direct solvers and they are always preferred for the solution of large 3D problems.
} 


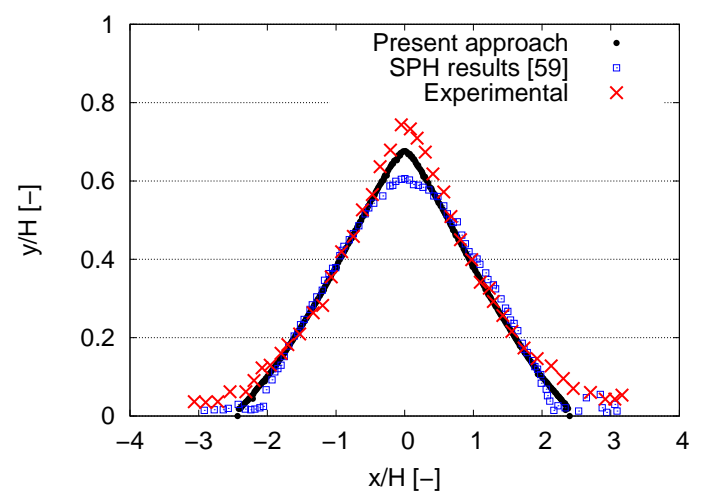

Figure 10: Granular column collapse. Free-surface position at $t^{*}=5.88$ obtained with the Exponential method and $\lambda=0.01 \mathrm{~s}^{-1}$. Comparison to experimental and numerical results [59].

The iterative linear solver used in this study is the so-called Biconjugate Gradient Stabilized (BiCGSTAB), one of most popular iterative schemes belonging to the family of Krylov subspace methods [54]. In all the examples, a maximum number of iterations of 5000 and a tolerance of $10^{-9}$ have been considered. Preconditioning techniques are generally used to improve the condition number of the linear system and, consequently, the convergence of the iterative method. In this work, the Incomplete-LU (ILU) [54] preconditioner is used.

The problem is first solved without using a preconditioner in order to highlight more clearly the conditioning problems induced by the standard $\mu(I)$ rheology. Table 1 collects the mean and the maximum iterations numbers obtained for three different meshes with the non-regularized method and the Exponential and Penalty regularizations. Three different values of the parameter $\lambda$ have been considered for each regularized method.

Several conclusions can be drawn from these results. First of all, the mesh refinement, and the consequent increasing of linear system size, produce an increment of iterations for all the methods analyzed. However, it is shown a clear difference between the regularized and non-regularized methods. Without regularization, the linear solver stops to converge at some point of the analysis, also when a coarse mesh is used (with the finest analyzed mesh, it is not even able to converge at the first computation step). On the other hand, a clear reduction of the linear solver iterations is obtained when one of the proposed regularization techniques is used. In particular, the higher is the regularization parameter $\lambda$, the smaller is the number of iterations. The results also show that the Exponential and Penalty methods behave similarly for the same values of $\lambda$, although the Exponential regularization gives a slightly smaller number of iterations than the Penalty one.

Figure 11 plots the iterations number at each computation step obtained with non-regularized $\mu(I)$-rheology and with the Penalty and Exponential meth- 


\begin{tabular}{|c|c|c|c|}
\hline Mesh size & Regularization & Mean iterations & Max iterations \\
\hline \hline \multirow{5}{*}{$0.005 \mathrm{~m}$} & None & 248.0 (until t*=1.48) & 5000 \\
\cline { 2 - 4 } & Penalty $\lambda=0.001 s^{-1}$ & 181.1 & 347 \\
& Penalty, $\lambda=0.01 s^{-1}$ & 67.8 & 132 \\
& Penalty, $\lambda=0.1 s^{-1}$ & 34.2 & 63 \\
\cline { 2 - 4 } & Exponential, $\lambda=0.001 s^{-1}$ & 161.9 & 339 \\
& Exponential, $\lambda=0.01 s^{-1}$ & 63.7 & 123 \\
& Exponential, $\lambda=0.1 s^{-1}$ & 34.6 & 61 \\
\hline \hline \multirow{5}{*}{$0.003 \mathrm{~m}$} & None & 369.6 (until t*=1.06) & 5000 \\
\cline { 2 - 4 } & Penalty, $\lambda=0.001 s^{-1}$ & 378.4 & 833 \\
& Penalty, $\lambda=0.01 s^{-1}$ & 132.5 & 314 \\
& Penalty, $\lambda=0.1 s^{-1}$ & 52.7 & 124 \\
\cline { 2 - 4 } & Exponential, $\lambda=0.001 s^{-1}$ & 332.2 & 791 \\
& Exponential, $\lambda=0.01 s^{-1}$ & 121.2 & 300 \\
& Exponential, $\lambda=0.1 s^{-1}$ & 53.0 & 133 \\
\hline \hline \multirow{5}{*}{$0.001 \mathrm{~m}$} & None & no convergence & 5000 \\
\cline { 2 - 4 } & Penalty, $\lambda=0.001 s^{-1}$ & 1855.1 & 3813 \\
& Penalty, $\lambda=0.01 s^{-1}$ & 500.3 & 1127 \\
& Penalty, $\lambda=0.1 s^{-1}$ & 161.1 & 435 \\
\cline { 2 - 4 } & Exponential, $\lambda=0.001 s^{-1}$ & 1591.9 & 1148 \\
& Exponential, $\lambda=0.01 s^{-1}$ & 475.4 & 366 \\
\cline { 2 - 4 } & Exponential, $\lambda=0.1 s^{-1}$ & 155.6 & \\
\hline
\end{tabular}

Table 1: Granular column collapse. Linear iterations using BiCGSTAB solver without preconditioner.

ods for $\lambda=0.001 \mathrm{~s}^{-1}$ and a mesh with size $0.005 \mathrm{~m}$. The graphs show that, when the non-regularized model is used, the iterations increase significantly after $t^{*}=1$ and, at $t^{*}=1.48$, the linear solver cannot converge before the prefixed limit of 5000 iterations. On the other hand, both Penalty and Exponential methods always maintain the linear iterations number small enough to proceed with the analysis.

Table 2 collects the mean and the maximum linear iterations obtained by the different methods, when also the ILU preconditioner is used. The results show that the preconditioner drastically reduces the linear iterations. It is important to note that, in the finest mesh case, despite the use of the preconditioner, the solver cannot convergence beyond $t^{*}=7.24$ if the non-regularized $\mu(I)$-rheology is used.

At that stage, the granular flow is close to reach the quiescent state, and the apparent viscosity, becoming extremely high, causes the deterioration of the linear system conditioning. On the contrary, the Exponential and Penalty regularizations are able to solve the granular flow even when it is practically at rest, also when a fine mesh is used.

Figure 12 shows the free-surface position at $t^{*}=5.88$ obtained with the nonregularized $\mu(I)$-rheology and the Penalty and Exponential regularizations with 


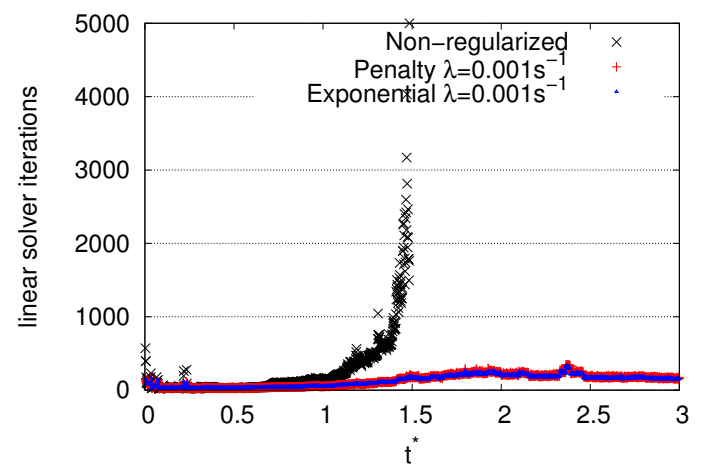

Figure 11: Granular column collapse. Maximum number of linear solver iterations at each time step. Linear solver: BiCGSTAB without preconditioner. Mesh size equal to $0.005 \mathrm{~m}$.

$\lambda=0.01 s^{-1}$. The differences among the three solutions are almost negligible and this confirms that the proposed regularized models are able to approximate accurately the standard $\mu(I)$-rheology.

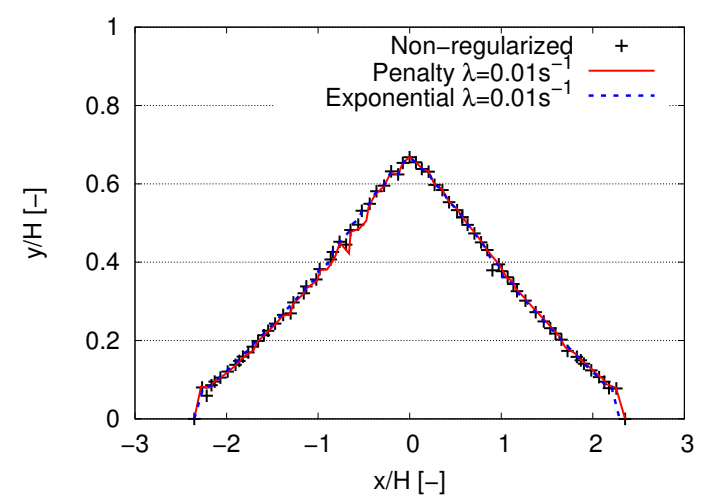

Figure 12: Granular column collapse. Free-surface position at $t^{*}=5.88$ given by regularized and non-regularized methods.

It is also interesting to analyze the effect of $\lambda$ on the accuracy of the numerical results. As already shown in Section 3, the larger is $\lambda$, the less accurate is the approximation of the original $\mu(I)$-curve (see Figures 5 and 6 ). In particular, $\lambda$ affects mainly the behavior of the granular mass when it is close to the state of rest. If a high value of $\lambda$ is chosen, the quiescent state cannot be reproduced accurately. Hence, although the conditioning of the linear system is improved by increasing the regularization parameter, $\lambda$ cannot been chosen arbitrarily large. To highlight this specific issue, in Figure 13 the evolution in time of the granular column height is given for the non-regularized method and for the 


\begin{tabular}{|c|c|c|c|}
\hline Mesh size & Regularization & Mean iterations & Max iterations \\
\hline \multirow{7}{*}{$0.005 \mathrm{~m}$} & None & 10.0 & 33 \\
\hline & Penalty, $\lambda=0.001 s^{-1}$ & 9.8 & 26 \\
\hline & Penalty, $\lambda=0.01 s^{-1}$ & 7.6 & 11 \\
\hline & Penalty, $\lambda=0.1 s^{-1}$ & 5.9 & 9 \\
\hline & Exponential, $\lambda=0.001 s^{-1}$ & 10.5 & 25 \\
\hline & Exponential, $\lambda=0.01 \mathrm{~s}^{-1}$ & 7.4 & 11 \\
\hline & Exponential, $\lambda=0.1 \mathrm{~s}^{-1}$ & 5.9 & 9 \\
\hline \multirow{7}{*}{$0.003 \mathrm{~m}$} & None & 25.4 & 164 \\
\hline & Penalty, $\lambda=0.001 s^{-1}$ & 18.6 & 30 \\
\hline & Penalty, $\lambda=0.01 \mathrm{~s}^{-1}$ & 11.8 & 29 \\
\hline & Penalty, $\lambda=0.1 s^{-1}$ & 7.1 & 20 \\
\hline & Exponential, $\lambda=0.001 s^{-1}$ & 18.0 & 27 \\
\hline & Exponential, $\lambda=0.01 \mathrm{~s}^{-1}$ & 11.4 & 18 \\
\hline & Exponential, $\lambda=0.1 \mathrm{~s}^{-1}$ & 7.0 & 11 \\
\hline \multirow{7}{*}{$0.001 \mathrm{~m}$} & None & $94.3\left(\right.$ until $\left.t^{*}=7.24\right)$ & 5000 \\
\hline & Penalty, $\lambda=0.001 s^{-1}$ & 59.6 & 91 \\
\hline & Penalty, $\lambda=0.01 s^{-1}$ & 38.0 & 60 \\
\hline & Penalty, $\lambda=0.1 \mathrm{~s}^{-1}$ & 20 & 40 \\
\hline & Exponential, $\lambda=0.001 s^{-1}$ & 60.1 & 86 \\
\hline & Exponential, $\lambda=0.01 \mathrm{~s}^{-1}$ & 38.5 & 52 \\
\hline & Exponential, $\lambda=0.1 s^{-1}$ & 19.2 & 25 \\
\hline
\end{tabular}

Table 2: Granular column collapse. Linear iterations using BiCGSTAB linear solver with $I L U$ preconditioner.

Exponential approximation considering three values of $\lambda^{4}$. The plot shows that the proposed method with small values of $\lambda$ is able to approximate accurately the non-regularized solution. On the other hand, for high values, such as $\lambda=$ $0.1 s^{-1}$, the regularized method diverges with respect to the non-regularized $\mu(I)$-rheology when the fluid is almost at rest.

\subsection{Flow of granular material over inclined planes}

In this section, the collapse of granular columns over inclined planes are reproduced with the Exponential regularization with $\lambda=0.01 \mathrm{~s}^{-1}$. The problem, taken from the experimental work [42], has been already studied with the $\mu(I)$ rheology in the visco-plastic model proposed in [33]. The experimental setup consists of a rectangular granular mass of height $h=14 \mathrm{~cm}$, length $r=20 \mathrm{~cm}$ and width $w=10 \mathrm{~cm}$, placed over a channel with variable inclination $\theta$, as shown in Figure 14a. The granular column is composed by glass beads $\left(d_{g}=0.7 \mathrm{~mm}\right.$,

\footnotetext{
${ }^{4}$ The same analysis has been done for the Penalty method, obtaining practically the same results than the Exponential method. However, for reasons of space the graphs referring to the Penalty method are not reported.
} 

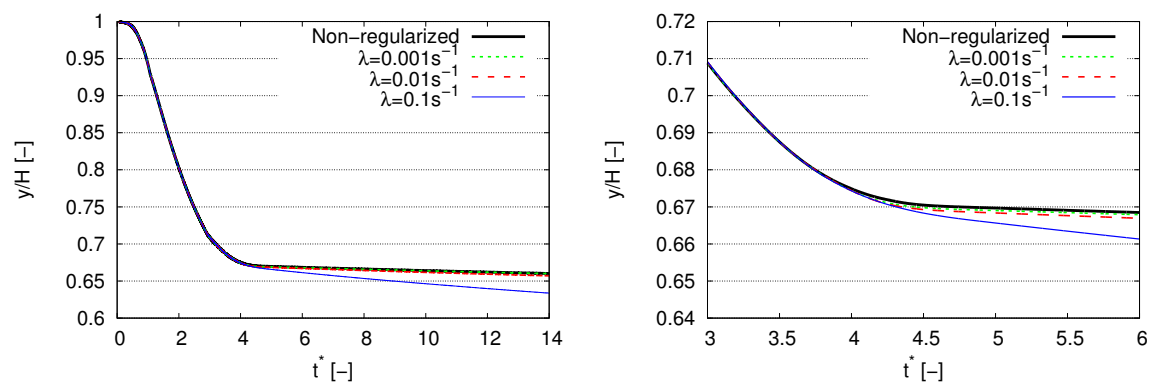

Figure 13: Granular column collapse. Normalized Y-coordinate for mesh size equal to $0.003 \mathrm{~m}$. Results for non-regularized method and Exponential regularization with three different $\lambda$ values.

(a) Initial geometry

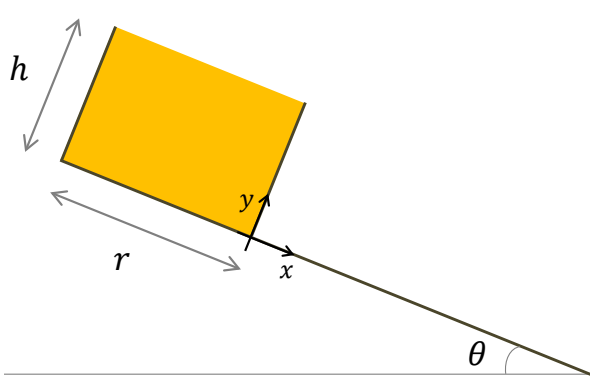

(b) Initial 3D mesh

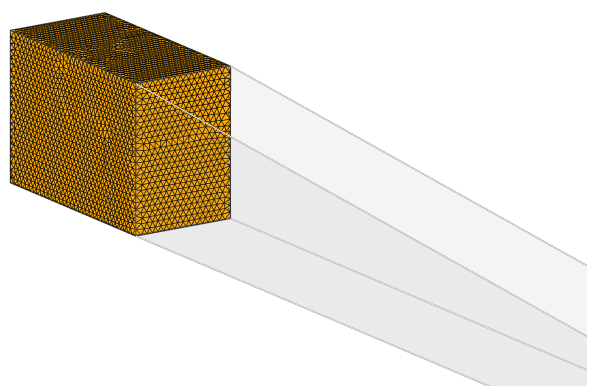

Figure 14: Flow of granular material over inclined planes. Initial geometry and 3D mesh.

$\rho_{g}=2500 \mathrm{~kg} / \mathrm{m}^{3}, \mu_{s}=0.38, \mu_{d}=0.64$ and $\left.I_{0}=0.279\right)$ and the bulk density has been considered fixed to $\rho=1550 \mathrm{~kg} / \mathrm{m}^{3}$.

The problem is first studied in $3 \mathrm{D}$ for $\theta=16^{\circ}$ to check if the $2 \mathrm{D}$ solution can correctly describe the flow in the middle of the channel and to show the applicability of the regularized $\mu(I)$-rheology to computational problems of large size. Next, other three different inclinations of the plane, namely $0^{\circ}, 10^{\circ}$ and $22^{\circ}$, are studied in $2 \mathrm{D}$.

As for the previous numerical example a dimensionless time $t^{*}=t / \sqrt{g / h}$ is used to plot the results.

\subsection{1. $3 D$ analysis for inclination angle $\theta=16^{\circ}$}

The case with the plane inclined of $\theta=16^{\circ}$ is first studied to compare 3D and $2 \mathrm{D}$ results. Finite element meshes composed by 7130 triangular and 322574 tetrahedral elements are used for the 2D and the 3D analysis, respectively. The employed 3D mesh is plotted in Figure 14b. Figure 15 shows the numerical results of the 3D analysis at different time instants. Moreover, Figure 16a 
(a) $t^{*}=1.256$

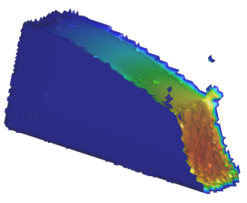

(c) $t^{*}=2.511$

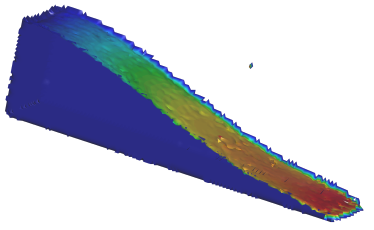

(e) $t^{*}=7.533$
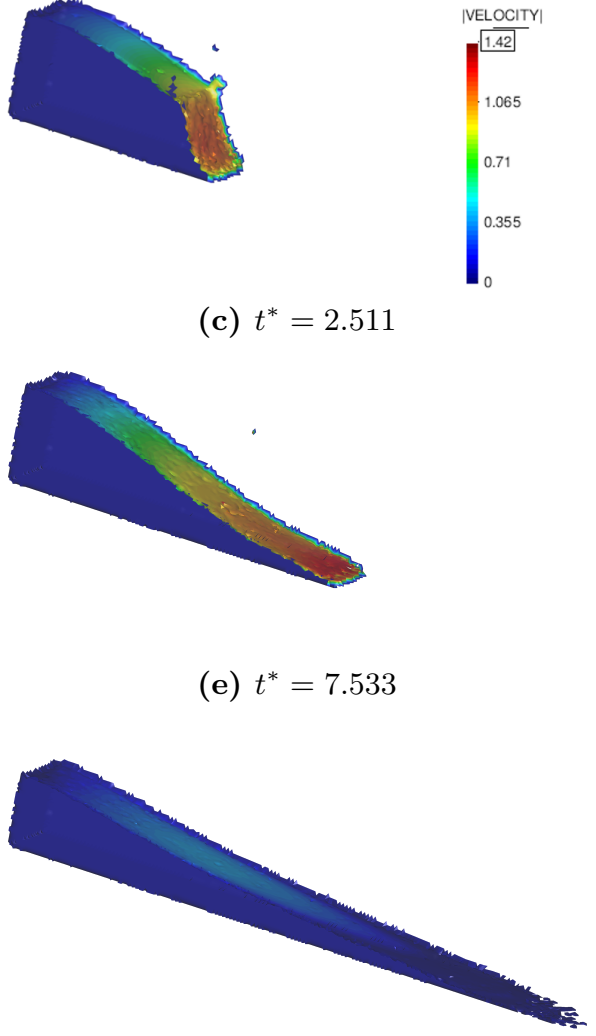

(b) $t^{*}=1.674$

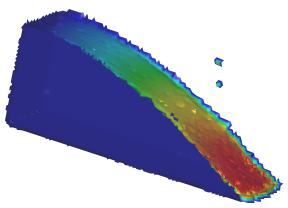

(d) $t^{*}=4.185$

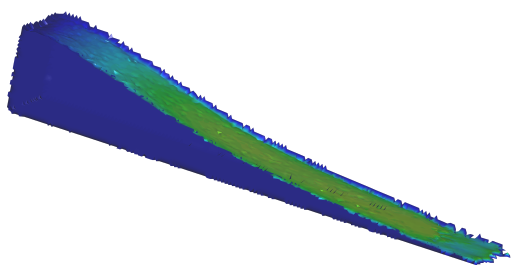

(f) $t^{*}=10.88$

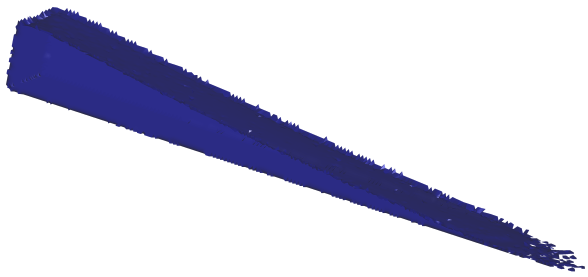

Figure 15: 3D flow of granular material over a plane with inclination angle $\theta=16^{\circ}$. Numerical results at six time instants.

plots the evolution of the normalized runout $x / h$ obtained experimentally and numerically in $2 \mathrm{D}$ and in 3D, being $x$ the distance covered by the granular flow along the inclined plane, as shown in Figure 14a. Finally, Figure 16b shows the free-surface configuration at the end of the analysis $\left(t^{*}=10.88\right)$.

Concerning the granular mass runout, both $2 \mathrm{D}$ and $3 \mathrm{D}$ simulations show a very good agreement with the experimental results (Figure 16a). On the other hand, some differences are exhibited in the final mass profile. While the 2D solution captures almost perfectly the final experimental configuration, the 3D results are not so accurate (Figure 16b). This may be due to the modeling of the interaction between the granular mass and the channel walls. As already mentioned, no-slip conditions have been considered and this may lead to overestimate the resistance to the flow exerted by the lateral walls. However, despite these small differences, the comparison with the experimental results can be considered absolutely satisfactory, in $2 \mathrm{D}$ as in $3 \mathrm{D}$.

In Figure 17 the number of iterations done by the linear solver at each 
(a) Runout evolution

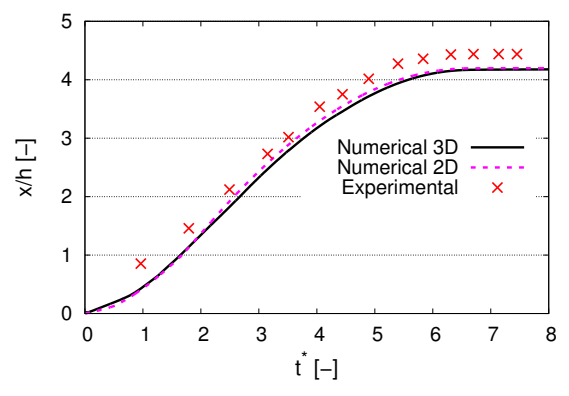

(b) Free-surface position

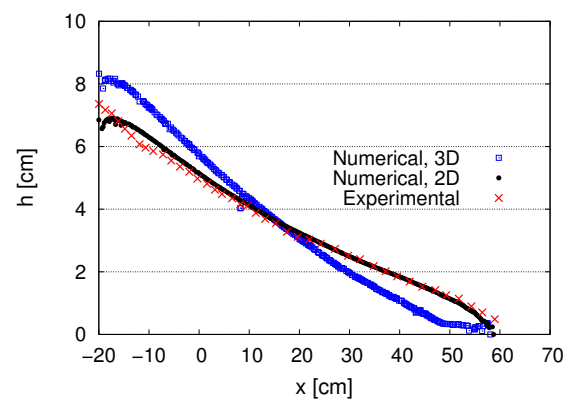

Figure 16: Flow of granular material over a plane with inclination angle $\theta=16^{\circ}$. (a) Normalized runout over dimensionless time. (b) Experimental [42] and numerical free-surface final position.

computational step of the 3D analysis is plotted. The graph shows that, despite the high number of elements, hence the large size of the linear system, the solver still needs a small number of iterations to converge. This shows the applicability of the proposed regularized model to real 3D cases.

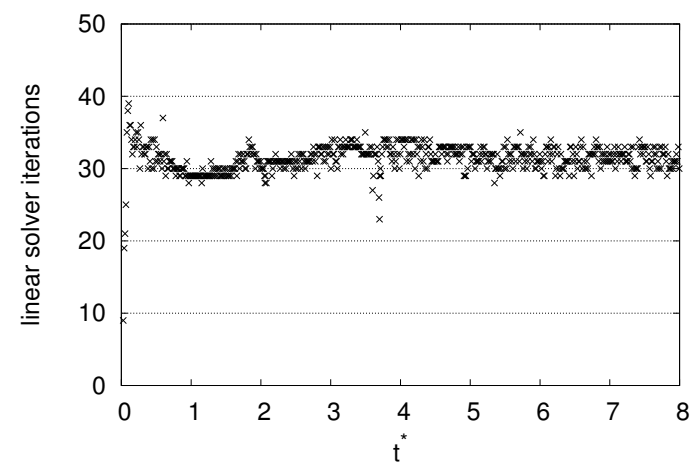

Figure 17: Flow of granular material over inclined planes $\left(\theta=16^{\circ}\right)$. Linear iterations of the $3 \mathrm{D}$ problem.

For comparison purposes, the 2D problem has been solved also using the non-regularized $\mu(I)$-rheology and the Penalty regularization $\left(\lambda=0.01 s^{-1}\right)$. Four different meshes have been considered (mean mesh sizes: $0.010 \mathrm{~m}, 0.0075 \mathrm{~m}$, $0.005 \mathrm{~m}$ and $0.003 \mathrm{~m}$ ) and the BiCGSTAB solver with $I L U$ preconditioner has been used. The number of linear iterations obtained by the different methods and meshes is given in Table 3 .

The results confirm the findings of the previous examples. The regularized methods reduce the number of iterations required by the solver to converge. 


\begin{tabular}{|c|c|c|c|}
\hline Mesh size & Regularization & Mean iterations & Max iterations \\
\hline \hline \multirow{3}{*}{$0.010 \mathrm{~m}$} & None & 13.0 & 28 \\
\cline { 2 - 4 } & Penalty, $\lambda=0.01 \mathrm{~s}^{-1}$ & 9.2 & 14 \\
& Exponential, $\lambda=0.01 \mathrm{~s}^{-1}$ & 9.2 & 14 \\
\hline \hline \multirow{3}{*}{$0.0075 \mathrm{~m}$} & None & 20.2 & 226 \\
\cline { 2 - 4 } & Penalty, $\lambda=0.01 \mathrm{~s}^{-1}$ & 12.8 & 21 \\
& Exponential, $\lambda=0.01 \mathrm{~s}^{-1}$ & 12.7 & 20 \\
\hline \hline \multirow{3}{*}{$005 \mathrm{~m}$} & None & 70.7 (until t* $=15.29)$ & 5000 \\
\cline { 2 - 4 } & Penalty, $\lambda=0.01 \mathrm{~s}^{-1}$ & 19.6 & 35 \\
& Exponential, $\lambda=0.01 \mathrm{~s}^{-1}$ & 19.1 & 31 \\
\hline \hline \multirow{3}{*}{$003 \mathrm{~m}$} & None & no convergence & - \\
\cline { 2 - 4 } & Penalty, $\lambda=0.01 \mathrm{~s}^{-1}$ & 34.2 & 58 \\
& Exponential, $\lambda=0.01 \mathrm{~s}^{-1}$ & 33.0 & 55 \\
\hline
\end{tabular}

Table 3: 2D flow of granular material over a plane with inclination angle $\theta=16^{\circ}$. Number of linear iterations using BiCGSTAB solver with $I L U$ preconditioner.

Without regularization the solver can only converge if coarse meshes are used, while, when finer meshes are employed, at a given point the convergence is lost. In particular, for the finest mesh (mean size $0.003 \mathrm{~m}$ ), the convergence is not even reached at the first step, whereas the regularized methods are still competitive (mean number of iterations around 35).

\subsection{2. $2 D$ analysis of different inclinations}

Three other plane inclinations $\left(0^{\circ}, 10^{\circ}\right.$ and $\left.22^{\circ}\right)$ have been investigated using the $2 \mathrm{D}$ model. Figures 18 and 19 show some snapshots of the numerical results for $\theta=0^{\circ}$ and $\theta=22^{\circ}$. The velocity contours are plotted over the deformed configurations.

Figure 20 shows the time evolution of the normalized runout for the four inclinations compared with experimental data. The plot shows a very good agreement of the numerical results to the experimental data for all the inclinations considered. This proves that the proposed numerical model is not only capable to predict the final runout, but also to reproduce accurately the evolution of the front position of the granular mass in time.

The final configurations of the granular mass are also compared with the experimental profiles. Figure 21 refers to $\theta=0^{\circ}$ and reports also the numerical results obtained numerically in [33] using a different method based on the $\mu(I)$ rheology. Results of Figure 22 refer to the case of $\theta=10^{\circ}$ and $\theta=22^{\circ}$, respectively. All the plots show a very good agreement between experimental and numerical results. The final granular mass profile is accurately predicted by the proposed regularized model.

\subsection{D simulation of the collapse of an cylindrical granular column.}

In this test the collapse of a cylinder of granular material presented in [39] is studied. The problem is solved using the Exponential regularization and 
(a) $t^{*}=0.5023$

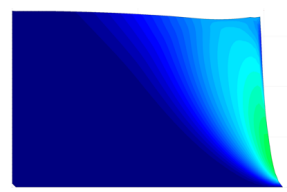

(c) $t^{*}=1.674$

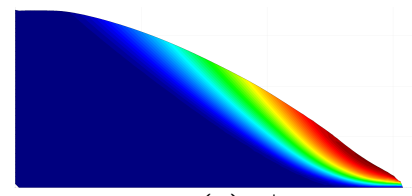

(e) $t^{*}=5.023$

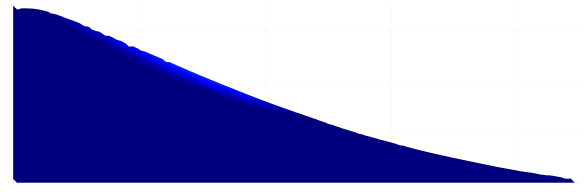

(b) $t^{*}=1.088$

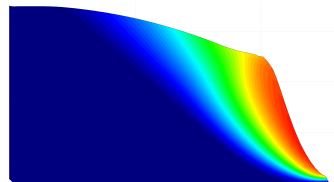

(d) $t^{*}=3.348$

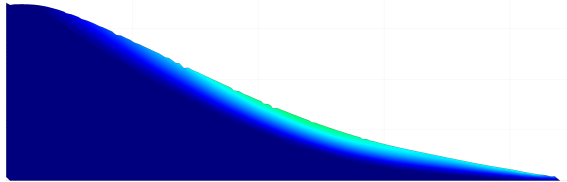

(f) $t^{*}=8.371$

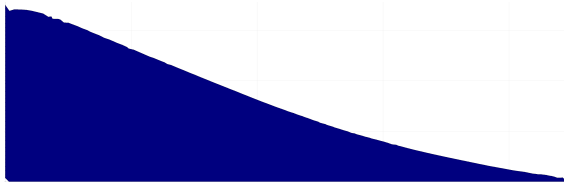

Figure 18: Flow of granular material over inclined planes $\left(\theta=0^{\circ}\right)$. Numerical results at six time instants.

$\lambda=0.01 \mathrm{~s}^{-1}$. The cylinder has initial height and radius $H=0.175 \mathrm{~m}$ and $R=0.035 \mathrm{~m}$, respectively. The initial mesh is composed by 248553 tetrahedra and the mean element size is $0.003 \mathrm{~m}$. The initial geometry and finite element mesh are plotted in Figure 23.

The grain properties $\left(d_{g}=2.0 \mathrm{~mm}\right.$ and $\rho_{g}=2500 \mathrm{~kg} / \mathrm{m}^{3}, \mu_{s}=0.4, \mu_{d}=0.7$ and $I_{0}=0.3$ ) are taken from [39]. The density of the bulk is kept fixed to $1550 \mathrm{~kg} / \mathrm{m}^{3}$ for all the analysis. As for the previous tests, no-slip conditions have been considered for the rigid horizontal plane.

Figure 24 collects some significant snapshots of the 3D simulation. While in Figure 25, the velocity and pressure contours are depicted on a vertical section.

After the instantaneous release of the granular column, three flow phases are easily recognizable. At the beginning, the top of the column is undergoing an almost free-fall regime (Figures 24a-24c and 25a-25d). After that, the grains move over an almost stationary deposit located at the core of the granular mass (heap regime [39]), as shown in Figures 24d and 25e-25f. Finally, before the stoppage, the flow, which just affects a narrow layer of the granular mass near to the free-surface, becomes mainly horizontal (Figures 24e-24g and 25g$25 j)$. These three phases characterize the collapse of granular columns with high aspect ratio $(H / R>2)$, as shown experimentally in [41] and numerically in [39].

In Figure 26 the evolution in time of the dimensionless radius variation $((r-R) / R)$ and residual height $(h / H)$ obtained with the proposed formulation is compared to the DEM results presented in [39]. The comparison shows a very good agreement between continuum and discrete numerical solutions. Note that between the initialization and the final sudden deceleration, the curve of Figure 
(a) $t^{*}=0.8371$

(b) $t^{*}=2.511$
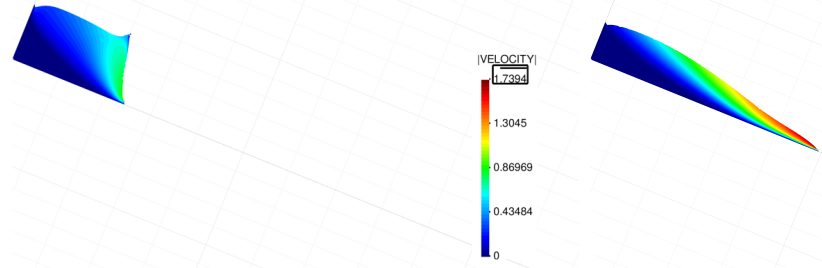

(c) $t^{*}=4.185$

(d) $t^{*}=8.371$

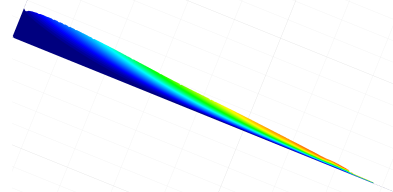

(e) $t^{*}=12.56$

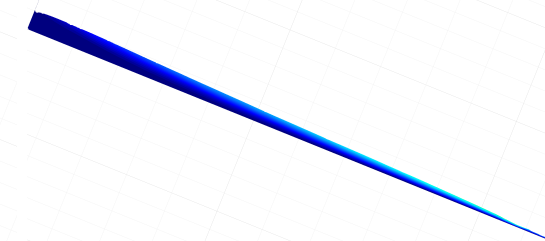

(f) $t^{*}=16.74$
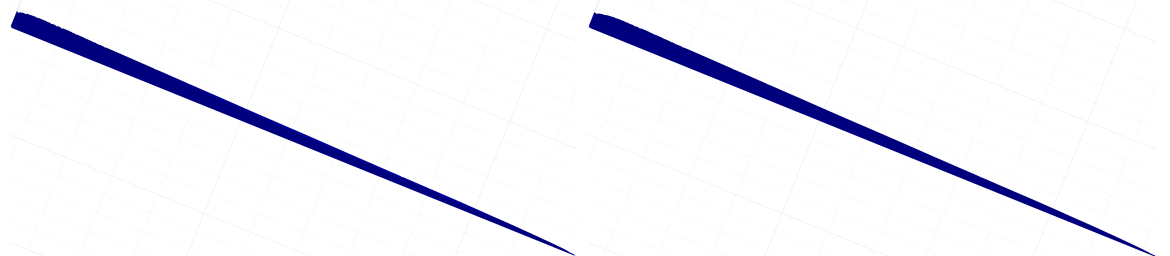

Figure 19: Flow of granular material over inclined planes $\left(\theta=22^{\circ}\right)$. Numerical results at six time instants.

26a shows a constant slope (between $0.05 \mathrm{~s}$ and $0.3 \mathrm{~s}$, approximately), as it is also found also experimentally in [41] where several collapses of granular columns are studied. The main discrepancy between continuum (PFEM) and discrete (DEM) results is exhibited at the final phase of the column collapse. Specifically, the DEM solution gives an higher final radius than the one predicted by this PFEM formulation. Note that this is analogous to what is obtained in [40], where the 2D collapse of granular columns is studied with both a continuum and a discrete method (in all the tests analyzed in [40], the DEM solution predicts a larger final diameter than the continuum method). Nevertheless, the comparison can be considered absolutely satisfactory and, although this is not considered within the scope of this article, the accuracy of these 3D results suggests the applicability of the $\mu(I)$-rheology also to multi-directional transient flows, as it was found in [39]. 


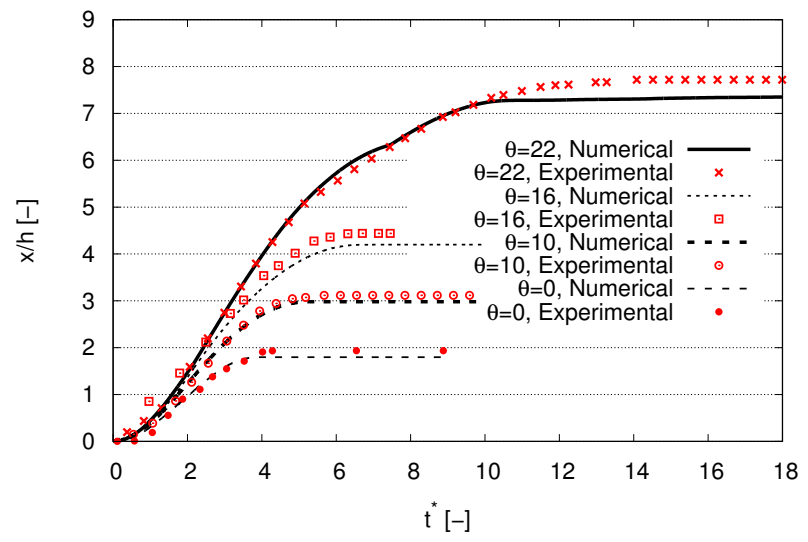

Figure 20: Flow of granular material over inclined planes. Evolution of normalized runout on dimensionless time. Comparison between experimental [42] and numerical $2 \mathrm{D}$ results.

\section{Conclusions}

In this work two regularized models of the $\mu(I)$-rheology have been presented and their application to complex 2D and 3D dense granular flows simulation has been shown. The so-called Exponential and Penalty regularizations are inspired by the Papanastasiou [48] and Bercovier-Engleman [6] methods typically used to approximate the Bingham law. The regularized $\mu(I)$-rheology has been implemented into a fluid dynamic framework based on the Particle Finite Element Method (PFEM).

To avoid the ill-conditioning of the linear system the proposed regularization strategies keep the apparent viscosity of the $\mu(I)$-rheology bounded. This approximation is performed without affecting the results of the standard $\mu(I)$ rheology.

The beneficial effects of the methods have been shown by analyzing $2 \mathrm{D}$ and 3D examples. The number of iterations required by the iterative linear solver to converge has been used as indicator of the conditioning of the linear system. From this study, several conclusions can be drawn. First of all, it has been shown that the standard $\mu(I)$-rheology leads to a such a ill-conditioned linear system that the iterative solver, without a preconditioner, is not able to converge neither when a very coarse mesh is used. Furthermore, even when a preconditioner is used, the linear solver diverges using finer meshes. On the other hand, both Exponential and Penalty regularizations guarantee a limited number of iterations, also when a fine mesh is used. Nevertheless, the use of a preconditioner is crucial in order to keep low the number of iterations also for the regularized models. The beneficial effect of the regularization parameter $\lambda$ has been also highlighted. The larger is $\lambda$, the better conditioned is the linear system. However, it has been shown that, for excessively large values of $\lambda$ (higher 


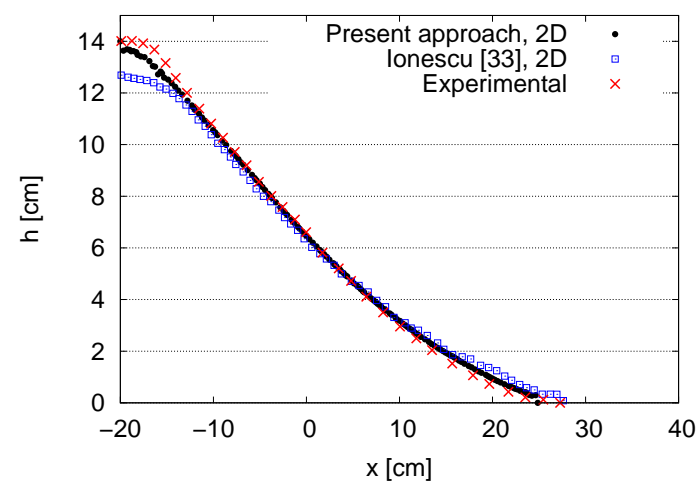

Figure 21: Flow of granular material over inclined planes $\left(\theta=0^{\circ}\right)$. Final free-surface position. Comparison to experimental [42] and other numerical [33] results.

(a) $\theta=10^{\circ}$

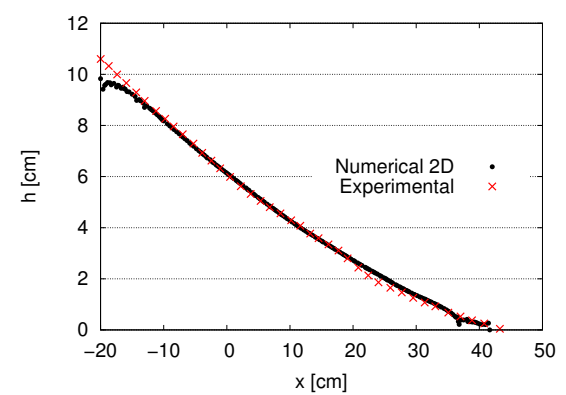

(b) $\theta=22^{\circ}$

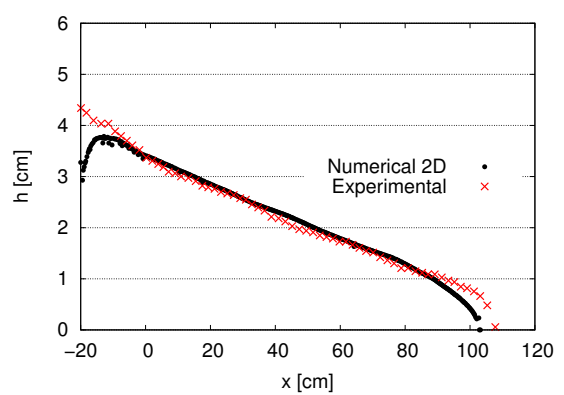

Figure 22: Flow of granular material over inclined planes. Experimental [42] and numerical free-surface final position for inclination angles $\theta=10^{\circ}$ and $\theta=22^{\circ}$.

than $0.01 \mathrm{~s}^{-1}$ ), the regularized solution diverges from the non-regularized one when the material is approaching the state of rest. It has been also shown that the Penalty and the Exponential methods behave similarly when the same value of the regularization parameter $\lambda$ is used, although a slight reduced number of iterations is obtained when the Exponential method is used. This motivated the use of the Exponential method for the second and the third test cases, where the regularized $\mu(I)$-rheology has been successfully applied to computationally demanding 3D problems.

Concerning the accuracy of the numerical simulations, a general good agreement with respect to the experimental tests has been obtained. The results are also in accordance with the numerical data obtained with different computational tools, such as the SPH and the DEM.

In conclusion, the numerical results have shown the computational benefits given by both proposed regularization methods, their accuracy with respect 


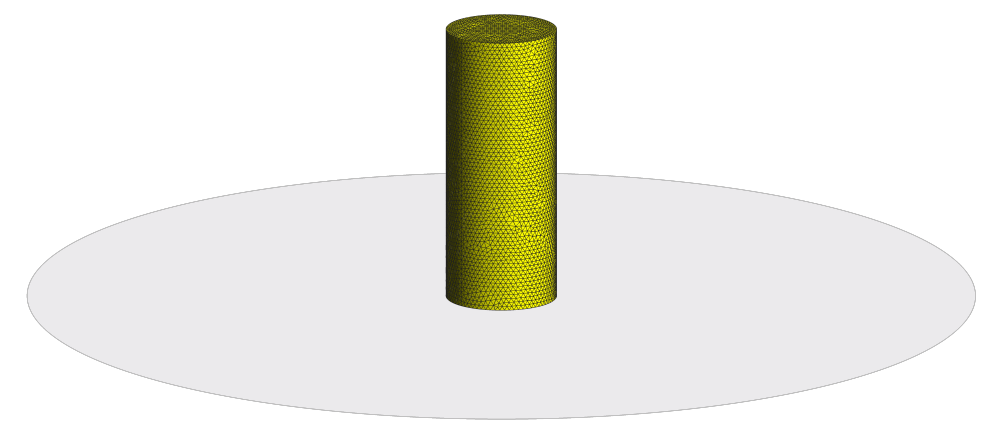

Figure 23: Collapse of an cylindrical granular column. Initial geometry and finite element mesh.

to the standard $\mu(I)$-rheology and the validity of this PFEM-based numerical formulation for the simulation of dense granular flow.

This work constitutes a first step in the numerical simulation of granular materials at different flow regimes. Next stages will be the extension to nonlocal granular rheology [37] and to other models which account for the transition between different phases [57].

\section{Acknowledgments}

The first author, beneficiary of an AXA Research Fund post-doctoral grant, acknowledges the company for its economic support. Both authors acknowledge Ilaria Iaconeta for the useful discussions.

\section{References}

[1] P. Aussillous, J. Chauchat, M. Pailha, M. Médale, and É. Guazzelli. Investigation of the mobile granular layer in bedload transport by laminar shearing flows. Journal of Fluid Mechanics, 736:594-615, 2013.

[2] M. Babic. Average balance equations for granular materials. International Journal of Engineering Science, 35 (5):523-548, 1997.

[3] T. Barker and J.M.N.T. Gray. Partial regularisation of the incompressible $\mu(\mathrm{I})$-rheology for granular flow. Journal of Fluid Mechanics, 828:5-32, 2017.

[4] T. Barker, D.G. Schaeffer, P. Bohorquez, and J.M.N.T. Gray. Well-posed and ill-posed behaviour of the $\mu(\mathrm{I})$-rheology for granular flow. Journal of Fluid Mechanics, 779:794-818, 2015.

[5] T. Barker, D.G. Schaeffer, M. Shearer, and J.M.N.T. Gray. Well-posed continuum equations for granular flow with compressibility and $\mu(\mathrm{I})$ rheology. Proceedings Mathematical, Physical, and Engineering Sciences, 473 (2201):20160846, 2017. 
(a) $t=0.05 \mathrm{~s}$

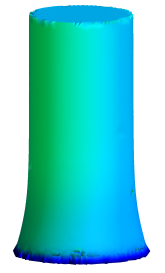

(c) $t=0.15 \mathrm{~s}$

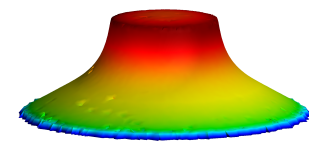

(e) $t=0.25 \mathrm{~s}$

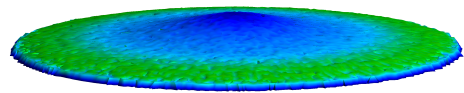

(g) $t=0.35 \mathrm{~s}$ (b) $t=0.10 \mathrm{~s}$

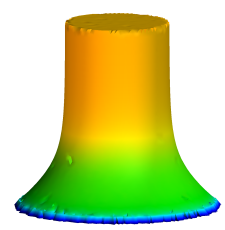

(d) $t=0.20 \mathrm{~s}$

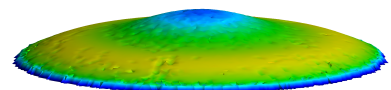

(f) $t=0.30 \mathrm{~s}$

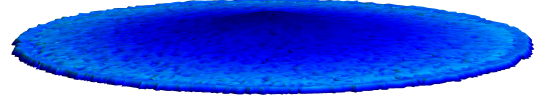

(h) $t=0.40 \mathrm{~s}$

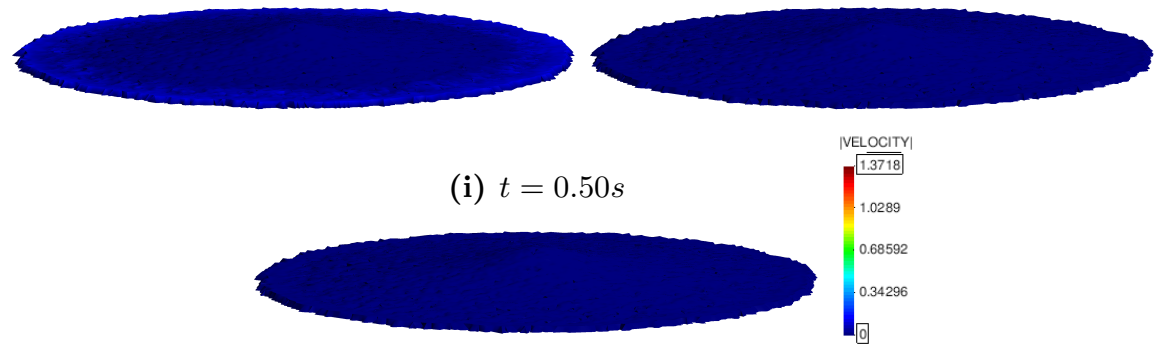

Figure 24: Collapse of an cylindrical granular column. Velocity contours plotted over the deformed $3 \mathrm{D}$ configuration.

[6] M. Bercovier and M. Engelman. A finite-element method for incompressible non-Newtonian flows. Journal of Computational Physics, 36 (3):313-326, 1980 .

[7] E.C. Bingham. Fluidity and Plasticity. McGraw-Hill, New York, 1922.

[8] F. Brezzi and M. Fortin. Mixed And Hybrid Finite Element Methods. Springer, New York, 1991.

[9] M.L. Cerquaglia, G. Deliége, R. Boman, V. Terrapon, and J.P. Ponthot. Free-slip boundary conditions for simulating free-surface incompressible flows through the particle finite element method. International Journal for Numerical Methods in Engineering, 110 (10):921-946, 2016. 
[10] J. Chauchat and M. Médale. A three-dimensional numerical model for incompressible two-phase flow of a granular bed submitted to a laminar shearing flow. Computer Methods in Applied Mechanics and Engineering, 199(9-12):439-449, 2010.

[11] J. Chauchat and M. Médale. A three-dimensional numerical model for dense granular flows based on the $\mu(\mathrm{I})$ rheology. Journal of Computational Physics, 256:696-712, 2014.

[12] W. Chen and T. Qiu. Numerical simulations for large deformation of granular materials using smoothed particle hydrodynamics method. International Journal of Geomechanics, 12(2):127-135, 2012.

[13] M. Cremonesi, F. Ferri, and U. Perego. A basal slip model for Lagrangian finite element simulations of 3D landslides. International Journal for $\mathrm{Nu}$ merical and Analytical Methods in Geomechanics, 41:30-53, 2017.

[14] M. Cremonesi and A. Frangi. Lagrangian finite element method for 3D compressible flow applications. Computer Methods in Applied Mechanics and Engineering, 311:374-392, 2016.

[15] M. Cremonesi, A. Frangi, and U. Perego. A Lagrangian finite element approach for the analysis of fluid-structure interaction problems. International Journal of Numerical Methods in Engineering, 84:610-630, 2010.

[16] G. B. Crosta, S. Imposimato, and D. Roddeman. Numerical modeling of 2D granular step collapse on erodible and nonerodible surface. Journal of Geophysical Research: Earth Surface, 114(F3).

[17] P.A. Cundall and O.D.L. Strack. A discrete numerical model for granular assemblies. Géotechnique, 29 (1):47-65, 1979.

[18] F. da Cruz, S. Emam, M. Prochnow, J.N. Roux, and F. Chevoir. Rheophysics of dense granular materials : Discrete simulation of plane shear flows. Physical Review E, 72 (2):021309, 2005.

[19] C. Davalos, J. Cante, J. Hernández, and X. Oliver. On the numerical modelling of granular material flows via the particle finite element method (PFEM). International Journal of Solids and Structures, 71:99-125, 2015.

[20] Gilles Daviet and Florence Bertails-Descoubes. Nonsmooth simulation of dense granular flows with pressure-dependent yield stress. Journal of NonNewtonian Fluid Mechanics, 234:15 - 35, 2016.

[21] D.C. Drucker and W. Prager. Soil mechanics and plastic analysis of limit design. Quarterly of Applied Mathematics, 10 (2):157-175, 1952.

[22] H. Edelsbrunner and E.P. Mucke. Three dimensional alpha shapes. ACM Trans Graphics, 13:43-72, 1999. 
[23] H. Edelsbrunner and T.S. Tan. An upper bound for conforming delaunay triangulations. Discrete and Computational Geometry, 10 (2):197:213, 1993.

[24] E.J. Fern and K. Soga. The role of constitutive models in MPM simulations of granular column collapses. Acta Geotechnica, 11(3):659-678, 2016.

[25] A. Franci and M. Cremonesi. On the effect of standard PFEM remeshing on volume conservation in free-surface fluid flow problems. Computational Particle Mechanics, 4 (3):331-343, 2017.

[26] A. Franci, E. Oñate, and J.M. Carbonell. On the effect of the bulk tangent matrix in partitioned solution schemes for nearly incompressible fluids. International Journal for Numerical Methods in Engineering, 102 (3-4):257$277,2015$.

[27] A. Franci, E. Oñate, and J.M. Carbonell. Unified Lagrangian formulation for solid and fluid mechanics and FSI problems. Computer Methods in Applied Mechanics and Engineering, 298:520-547, 2016.

[28] W.H. Herschel and R. Bulkley. Konsistenzmessungen von gummibenzollösungen. Kolloid-Zeitschrift, 39 (4):291-300, 1926.

[29] I. Iaconeta, A. Larese, R. Rossi, and Z. Guo. Comparison of a material point method and a Galerkin meshfree method for the simulation of cohesivefrictional materials. Materials, 10 (10):1150, 2017.

[30] S.R. Idelsohn, N. Calvo, and E. Oñate. Polyhedrization of an arbitrary point set. Computer Methods in Applied Mechanics and Engineering, 92 (22-24):2649-2668, 2003.

[31] S.R. Idelsohn, J. Marti, A. Limache, and E. Oñate. Unified Lagrangian formulation for elastic solids and incompressible fluids: Applications to fluid-structure interaction problems via the PFEM. Computer Methods In Applied Mechanics And Engineering, 197:1762-1776, 2008.

[32] S.R. Idelsohn, E. Oñate, and F. Del Pin. The particle finite element method: a powerful tool to solve incompressible flows with free-surfaces and breaking waves. International Journal for Numerical Methods in Engineering, 61:964-989, 2004.

[33] I.R. Ionescu, A. Mangeney, F. Bouchute, and O. Roche. Viscoplastic modeling of granular column collapse with pressure-dependent rheology. Journal of Non-Newtonian Fluid Mechanics, 219:1-18, 2015.

[34] I. Iordanoff and M.M. Khonsari. Granular lubrication: Toward an understanding of the transition between kinetic and quasi-fluid regime. Journal of Tribology, 126 (1):137-145, 2004. 
[35] P. Jop. Rheological properties of dense granular flows. Comptes Rendus Physique, 16:62-72, 2015.

[36] P. Jop, Y. Forterre, and O. Pouliquen. A constitutive law for dense granular flows. Nature, 441:727-730, 2006.

[37] K. Kamrin and G. Koval. Nonlocal constitutive relation for steady granular flow. Physical Review Letter, 108:178301, 2012.

[38] T. Karlsson, M. Klisinski, and K. Runesson. Finite element simulation of granular material flow in plane silos with complicated geometry. Powder Technology, 99(1):29 - 39, 1998.

[39] L. Lacaze and R.R. Kerswell. Axisymmetric granular collapse: A transient 3D flow test of viscoplasticity. Physical Review Letter, 102:108305, 2009.

[40] P.Y. Lagree, L. Staron, and S. Popinet. The granular column collapse as a continuum: validity of a two-dimensional Navier-Stokes model with a $\mu(\mathrm{I})$-rheology. Journal of Fluid Mechanics, 686:378-408, 2011.

[41] G. Lube, H. E. Huppert, S.J. Sparks, and M.A. Hallworth. Axisymmetric collapses of granular columns. Journal of Fluid Mechanics, 508:175-199, 2004.

[42] A. Mangeney, O. Roche, O. Hungr, N. Mangold, G. Faccaroni, and A. Lucas. Erosion and mobility in granular collapse over sloping beds. Journal of Geophysical Research, 115:F03040, 2010.

[43] GDR MiDi. On dense granular flows. The European Physical Journal E, 14:341-365, 2004.

[44] E. Oñate. Derivation of stabilized equations for advective-diffusive transport and fluid flow problems. Computer methods in applied mechanics and engineering, 151:233-267, 1998.

[45] E. Oñate, A. Franci, and J.M. Carbonell. Lagrangian formulation for finite element analysis of quasi-incompressible fluids with reduced mass losses. International Journal for Numerical Methods in Fluids, 74 (10):699-731, 2014.

[46] E. Oñate, S.R. Idelsohn, M.A. Celigueta, and R. Rossi. Advances in the particle finite element method for the analysis of fluid-multibody interaction and bed erosion in free surface flows. Computer methods in applied mechanics and engineering, 197 (19-20):1777-1800, 2008.

[47] E. Oñate, S.R. Idelsohn, F. Del Pin, and R. Aubry. The particle finite element method. An overview. International Journal for Computational Methods, 1:267-307, 2004.

[48] T.C. Papanastasiou. Flows of materials with yield. Journal of Rheology, 31:385-404, 1987. 
[49] O. Pouliquen. Scaling laws in granular flows down rough inclined planes. Physics of Fluids, 11:542-548, 1999.

[50] O. Pouliquen and Y. Forterre. Friction law for dense granular flows: application to the motion of a mass down a rough inclined plane. Journal of Fluid Mechanics, 453:133-151, 2002.

[51] I. De Pouplana and E. Oñate. A FICbased stabilized mixed finite element method with equal order interpolation for solid-pore fluid interaction problems. International Journal for Numerical and Analytical Methods in Geomechanics, 41:110-134, 2017.

[52] T. Revil-Baudard and J. Chauchat. A two-phase model for sheet flow regime based on dense granular flow rheology. Journal of Geophysical Research: Oceans, 118(2):619-634, 2013.

[53] P. Ryzhakov, E. Oñate, and S.R. Idelsohn. Improving mass conservation in simulation of incompressible flows. International Journal of Numerical Methods in Engineering, 90:1435-1451, 2012.

[54] Y. Saad. Iterative methods for sparse linear systems. SIAM, Philadelphia, 2nd Edition, 2003.

[55] P. Saramito and A. Wachs. Progress in numerical simulation of yield stress fluid flows. Rheologica Acta, 56(3):211-230, 2017.

[56] S.B. Savage. The mechanics of rapid granular flows. Advances in Applied Mechanics, 24:289-366, 1984.

[57] D. Vescovi, D. Berzi, and C. di Prisco. Fluid-solid transition in unsteady, homogeneous, granular shear flows. Granular Matter, 20(2):27, Mar 2018.

[58] T. Weinhart, C. Labra, S. Luding, and J.Y. Ooi. Influence of coarsegraining parameters on the analysis of dem simulations of silo flow. Powder Technolgy, 293:138-148, 2016.

[59] T. Xu, Y.C. Jin, Y.C. Tai, and C.H. Lu. Simulation of velocity and shear stress distributions in granular column collapses by a mesh-free method. Journal of Non-Newtonian Fluid Mechanics, 247:146-164, 2017.

[60] X. Zhang, K. Krabbenhoft, D.M. Pedroso, A.V. Lyamin, D. Sheng, M. Vicente da Silva, and D. Wang. Particle finite element analysis of large deformation and granular flow problems. Computer and Geotechnics, 54:133$142,2013$.

[61] X. Zhang, K. Krabbenhoft, and D. Sheng. Particle finite element analysis of the granular column collapse problem. Granular Matter, 16:609-619, 2014. 
(a) Velocity field at $t=0.05 \mathrm{~s}$

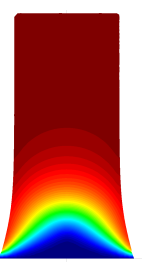

(c) Velocity field at $t=0.10 \mathrm{~s}$

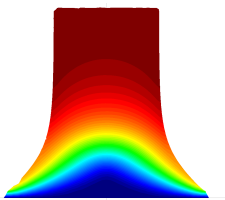

(e) Velocity field at $t=0.20 \mathrm{~s}$

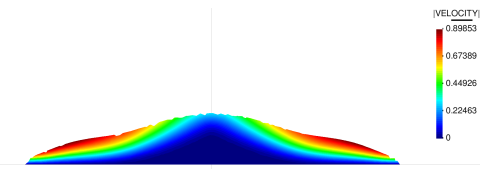

(g) Velocity field at $t=0.25 \mathrm{~s}$

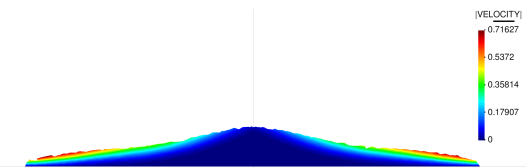

(i) Velocity field at $t=0.30 \mathrm{~s}$

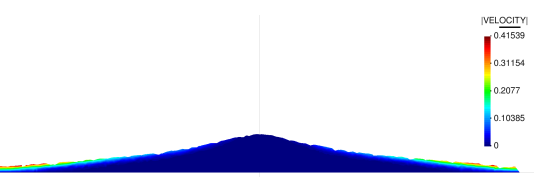

(b) Pressure field at $t=0.05 \mathrm{~s}$
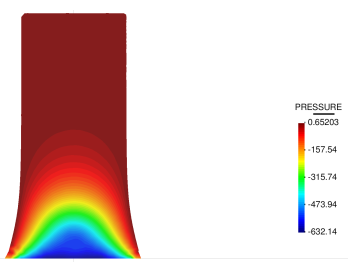

(d) Pressure field at $t=0.10 \mathrm{~s}$

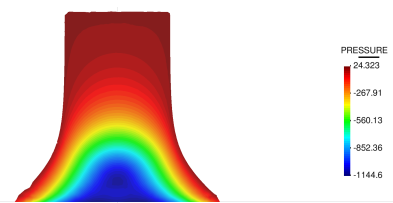

(f) Pressure field at $t=0.20 \mathrm{~s}$

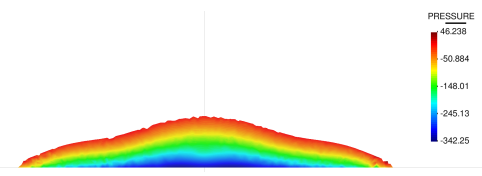

(h) Pressure field at $t=0.25 \mathrm{~s}$

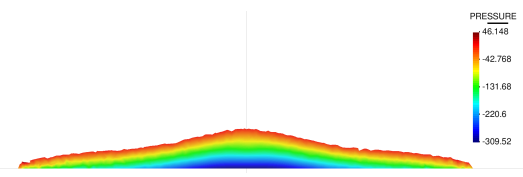

(j) Pressure field at $t=0.30 \mathrm{~s}$

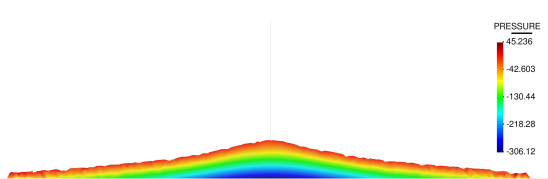

Figure 25: Collapse of an cylindrical granular column. Velocity and pressure fields plotted over a vertical section at different time instants. 
(a)

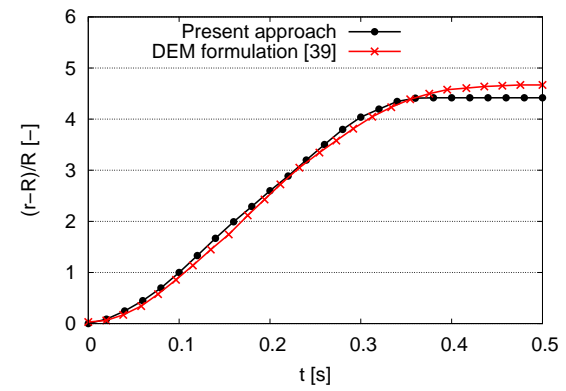

(b)

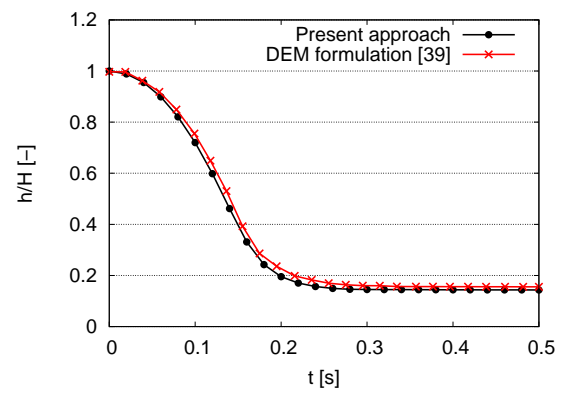

Figure 26: Collapse of an cylindrical granular column. Time evolution of dimensionless slump and height. Comparison to DEM formulation [39]. 